\title{
Excitonic Splitting and Vibronic Coupling Analysis of the Meta-Cyanophenol Dimer
}

Franziska A Balmer, Sabine Kopec, Horst Koeppel, and Samuel Leutwyler

J. Phys. Chem. A, Just Accepted Manuscript • DOI: 10.1021/acs.jpca.6b10416 • Publication Date (Web): 06 Dec 2016

Downloaded from http://pubs.acs.org on December 10, 2016

\section{Just Accepted}

"Just Accepted" manuscripts have been peer-reviewed and accepted for publication. They are posted online prior to technical editing, formatting for publication and author proofing. The American Chemical Society provides "Just Accepted" as a free service to the research community to expedite the dissemination of scientific material as soon as possible after acceptance. "Just Accepted" manuscripts appear in full in PDF format accompanied by an HTML abstract. "Just Accepted" manuscripts have been fully peer reviewed, but should not be considered the official version of record. They are accessible to all readers and citable by the Digital Object Identifier (DOI®). "Just Accepted" is an optional service offered to authors. Therefore, the "Just Accepted" Web site may not include all articles that will be published in the journal. After a manuscript is technically edited and formatted, it will be removed from the "Just Accepted" Web site and published as an ASAP article. Note that technical editing may introduce minor changes to the manuscript text and/or graphics which could affect content, and all legal disclaimers and ethical guidelines that apply to the journal pertain. ACS cannot be held responsible for errors or consequences arising from the use of information contained in these "Just Accepted" manuscripts. 


\title{
Excitonic Splitting and Vibronic Coupling Analysis
}

\section{of the meta-Cyanophenol Dimer}

\author{
Franziska A. Balmer ${ }^{\dagger}$ Sabine Kopec,${ }^{\ddagger}$ Horst Köppel, ${ }^{\ddagger}$ and Samuel Leutwyler ${ }^{*}$, \\ Department of Chemistry and Biochemistry, University of Bern, Freiestrasse 3, CH-3012 Bern, \\ Switzerland, and Theoretische Chemie, Physikalisch-Chemisches Institut, Universität Heidelberg, \\ Im Neuenheimer Feld 229, D-69120 Heidelberg, Germany. \\ E-mail: leutwyler@dcb.unibe.ch
}

\begin{abstract}
The $S_{1} / S_{2}$ splitting of the meta-cyanophenol dimer, $(\mathrm{mCP})_{2}$, and the delocalization of its excitonically coupled $S_{1} / S_{2}$ states are investigated by mass-selective two-color resonant two-photon ionization and dispersed fluorescence spectroscopy, complemented by a theoretical vibronic coupling analysis based on correlated $a b$ initio calculations at the approximate coupled cluster CC2 and SCS-CC2 levels. The calculations predict three close-lying groundstate minima of $(\mathrm{mCP})_{2}$ : The lowest is slightly Z-shaped $\left(C_{i}\right.$-symmetric), the second-lowest is $<5 \mathrm{~cm}^{-1}$ higher and planar $\left(C_{2 h}\right)$. The vibrational ground state is probably delocalized over both minima. The $S_{0} \rightarrow S_{1}$ transition of $(\mathrm{mCP})_{2}$ is electric-dipole allowed $\left(A_{g} \rightarrow A_{u}\right)$, while the $S_{0} \rightarrow S_{2}$ transition is forbidden $\left(A_{g} \rightarrow A_{g}\right)$. Breaking the inversion symmetry by ${ }^{12} \mathrm{C} /{ }^{13} \mathrm{C}$ - or H/D-substitution renders the $S_{0} \rightarrow S_{2}$ transition partially allowed; the excitonic contribution to the $S_{1} / S_{2}$ splitting is $\Delta_{\text {exc }}=7.3 \mathrm{~cm}^{-1}$. Additional isotope-dependent contributions arise from
\end{abstract}

\footnotetext{
${ }^{*}$ To whom correspondence should be addressed

${ }^{\dagger}$ Department of Chemistry and Biochemistry, University of Bern, Freiestrasse 3, CH-3012 Bern, Switzerland

¥Theoretische Chemie, Physikalisch-Chemisches Institut, Universität Heidelberg, Im Neuenheimer Feld 229, D69120 Heidelberg, Germany. 
the changes of the $m$-cyanophenol zero-point vibrational energy upon electronic excitation, which are $\Delta_{\text {iso }}\left({ }^{12} \mathrm{C} /{ }^{13} \mathrm{C}\right)=3.3 \mathrm{~cm}^{-1}$ and $\Delta_{\text {iso }}(\mathrm{H} / \mathrm{D})=6.8 \mathrm{~cm}^{-1}$. Only partial localization of the exciton occurs in the ${ }^{12} \mathrm{C} /{ }^{13} \mathrm{C}$ and H/D substituted heterodimers. The SCS-CC2 calculated excitonic splitting is $\Delta_{e l}=179 \mathrm{~cm}^{-1}$; when multiplying this with the vibronic quenching factor $\Gamma_{\text {vibron }}^{\text {exp }}=0.043$, we obtain an exciton splitting $\Delta_{\text {vibron }}^{\text {exp }}=7.7 \mathrm{~cm}^{-1}$, which agrees very well with the experimental $\Delta_{e x c}=7.3 \mathrm{~cm}^{-1}$. The semiclassical exciton hopping times range from $3.2 \mathrm{ps}$ in $(\mathrm{mCP})_{2}$ to $5.7 \mathrm{ps}$ in the heterodimer $(\mathrm{mCP}-h) \cdot(\mathrm{mCP}-d)$. A multimode vibronic coupling analysis is performed encompassing all the vibronic levels of the coupled $S_{1} / S_{2}$ states from the $\mathrm{v}=0$ level to $600 \mathrm{~cm}^{-1}$ above. Both linear and quadratic vibronic coupling schemes were investigated to simulate the $S_{0} \rightarrow S_{1} / S_{2}$ vibronic spectra, those calculated with the latter scheme agree better with experiment.

\section{Introduction}

Excitonic interactions between chromophores play an important role for electronic energy transfer in a wide range of photochemical and biological systems such as conjugated polymers, molecular crystals and photosynthetic light-harvesting complexes. ${ }^{1-8}$ Gas-phase hydrogen-bonded molecular dimers can be used to investigate these interactions since they are representative of larger systems, but are still small enough to yield well-resolved vibronic spectra and allow high-level ab initio calculations and vibronic coupling treatments. We have been studying the excitonic $S_{0} \rightarrow S_{1} / S_{2}$ splittings in rigid, doubly H-bonded aromatic dimers such as (2-pyridone) $)_{2}$, (2-aminopyridine) $)_{2}$, (benzoic acid $)_{2}$, (benzonitrile $)_{2}$ and (ortho-cyanophenol $)_{2}$ by mass- and isotope-selective two-color resonant two-photon ionization $(2 \mathrm{C}-\mathrm{R} 2 \mathrm{PI})$ spectroscopy with vibronic coupling calculations. ${ }^{9-18}$ These dimers are all inversion-symmetric, which implies that the $S_{1}$ excited states of their constituent monomers $A$ and $B$ are degenerate at large intermolecular distance. When the doubly H-bonded dimer is formed, the intermolecular coupling splits the monomer states into two nondegenerate excitonic states, one of which is symmetric $(g)$ and the other antisymmetric $(u)$, rendering the $S_{0} \rightarrow S_{1} / S_{2}$ electronic transitions forbidden and allowed, respectively. Isotopic substitution 
breaks the inversion symmetry of the dimer sufficiently to render the forbidden excitonic transition allowed, enabling to experimentally determine the $S_{1} \leftrightarrow S_{2}$ excitonic splitting. ${ }^{9-18}$

We have previously shown that the coupling of the $S_{1}$ and $S_{2}$ states to excited-state intra- and intermolecular vibrations has a substantial impact on the magnitude of the excitonic energy splittings. ${ }^{13,16}$ The observed splittings are up to 40 times smaller than the electronic Davydov splittings that are predicted by $a b$ initio calculations. ${ }^{9-18}$ This so-called "vibronic quenching" of the electronic excitonic splitting can be explained by taking the vibrational normal modes into account within Förster's perturbation theory approach ${ }^{19}$ and the Fulton-Gouterman model. ${ }^{20,21}$ Applying the vibronic quenching factor $\Gamma_{\text {vibron }}$ to the calculated Davydov splitting resulted in vibronic splittings very close to the experimentally observed $S_{1} / S_{2}$ splittings. ${ }^{13,15,17}$

Here, we combine experimental spectroscopic investigations, ab initio calculations and vibroniccoupling theory treatments to explain the $S_{1} / S_{2}$ state excitonic splitting and vibronic band structure of the meta-cyanophenol dimer, $(\mathrm{mCP})_{2}$. In contrast to the previously studied doubly H-bonded dimers, ${ }^{9-18}$ the $S_{0} \rightarrow S_{1}$ transition of $(\mathrm{mCP})_{2}$ is fully allowed, while the $S_{0} \rightarrow S_{2}$ transition is forbidden. This is due to the orientation of the $\mathrm{mCP}$ monomer electronic transition dipole moments, relative to the inter-monomer vector $R_{A B}$ between the centers of mass of $A$ and $B$, see Figure 1 .

Zehnacker and co-workers have previously studied $\mathrm{mCP}$ and $(\mathrm{mCP})_{2}$ by laser-induced fluorescence and fluorescence dip-IR spectroscopy. ${ }^{22}$ They observed both the cis- and trans-mCP rotamers, whose $\mathrm{OH}$ groups are oriented towards and away from the $\mathrm{CN}$ group. They reported that the meta-cyanophenol dimer, $(\mathrm{mCP})_{2}$, is dominantly formed by the cis-mCP rotamer, which can form two antiparallel $\mathrm{OH} \cdots \mathrm{N}$ hydrogen bonds, while the trans-mCP dimer, which can only form a single H-bond, was not observed experimentally. ${ }^{22}$ They proposed vibronic assignments of the observed bands and identified several fundamental transitions of non-totally-symmetric vibrations. In $C_{i}$-symmetry, these transitions would not be allowed. Hence, Zehnacker and co-workers concluded a loss of symmetry upon excitation that renders the fundamental transitions of non-totally symmetric vibrations allowed; the loss of symmetry was thought to cause a localization of the electronic excitation on one of the monomers. ${ }^{22}$ 
Below, we present the mass-selected two-color resonant two-photon ionization spectra of the homodimers $(\mathrm{mCP})_{2}$ and $(\mathrm{mCP}-d)_{2}$, and of the singly isotopically substituted $(\mathrm{mCP}) \cdot\left(\mathrm{mCP}-{ }^{13} \mathrm{C}\right)$ and $(\mathrm{mCP}-h) \cdot(\mathrm{mCP}-d)$ heterodimers. From the latter two spectra we determine the $S_{1} / S_{2}$ splittings and the contribution of the ${ }^{12} \mathrm{C} /{ }^{13} \mathrm{C}$ and H/D isotopic mass changes on the splitting, similar to (benzoic acid $)_{2}$ and (benzonitrile $)_{2} \cdot{ }^{14,15}$ A computational investigation of the $(\mathrm{mCP})_{2}$ ground state using correlated $a b$ initio methods reveals four different minimum-energy structures. The normal modes of the two lowest-energy structures form the basis for the following vibronic coupling treatment and band assignments in the spectrum of $(\mathrm{mCP})_{2}$. The linear and quadratic vibronic coupling schemes are employed ${ }^{23}$ to simulate the theoretical vibronic spectra. The influence of the vibronic coupling on the excitonic energy splitting is discussed and the question of the localization of the excited states addressed. While the $S_{1} / S_{2}$ coupled states are completely delocalized in the $(\mathrm{mCP})_{2}$ and (mCP- $d)_{2}$ homodimers, asymmetric isotopic substitution partially localizes the two electronic transitions. Using the effective-mode approximation introduced by Köppel and co-workers, ${ }^{24}$ we show that the double-minimum character of the lower excited state of the homodimer does not imply a localization of the excitation as proposed by Zehnacker and co-workers. ${ }^{22}$

\section{Theoretical Framework for Multimode Vibronic Coupling}

Recently, Köppel and co-workers have extended the analysis from the $S_{1} / S_{2}$ splitting to simulating the six-dimensional vibronically coupled spectra of (o-cyanophenol $)_{2}$ and (2-pyridone) $)_{2}$, and compared them to the $2 \mathrm{C}$-R2PI spectra. ${ }^{16,18}$ The representation of the vibronic coupling in the framework of the Fulton-Gouterman model ${ }^{20,21}$ was seen to be insufficient. Instead they adopted the more general linear and quadratic vibronic coupling schemes. ${ }^{16,18,23}$ These naturally include certain important intermolecular normal modes in the analysis, resulting in convincing simulations of the $S_{0} \rightarrow S_{1} / S_{2}$ vibronic spectra that were in good agreement with experiment. ${ }^{16,18}$ Other extensions of the Fulton-Gouterman model to vibronic coupling in excitonic systems have been made, see, for example, the application to bichromophores by Slipchenko, Zwier and co-workers. ${ }^{25-27}$ 


\subsection{The Multimode Vibronic Coupling Model}

The $S_{0} \rightarrow S_{1} / S_{2}$ vibronic spectrum of $(\mathrm{mCP})_{2}$ is investigated by means of the multimode vibronic coupling model described in ref. 23, including 12 vibrational modes, of which seven are totallysymmetric $\left(a_{g}\right)$ and five are non-totally symmetric $\left(a_{u}\right)$. This model has been applied before to (ortho-cyanophenol $)_{2}$ and (2-pyridone) $)_{2}$, and is there explained in detail. ${ }^{16,18}$ The Hamiltonian is constructed in a diabatic basis with a diagonal nuclear kinetic energy operator $T_{N}$ and a coupling matrix $\mathbf{W}$ emerging from the potential energy part.

$$
H=T_{N} \mathbf{1}+\mathbf{W}(\mathbf{Q})
$$

The coupling matrix elements are smooth functions of the dimensionless normal mode coordinates $Q_{i}$, and are expanded in a Taylor series around the ground-state equilibrium geometry $Q_{0}=0$. To obtain the linear (LVC) and quadratic (QVC) vibronic coupling models, the Taylorseries is truncated after the first and second order term, respectively. ${ }^{18,23}$

Coupling constants are the building blocks of the coupling matrix and are restricted by symmetry selection rules, determining which vibrational normal modes are relevant in the diagonal and off-diagonal elements. Vibrational modes with irreducible representation $\Gamma_{Q}$ can couple electronic states 1 and 2 with corresponding irreducible representations $\Gamma_{1}$ and $\Gamma_{2}$ only if their direct product comprises the totally symmetric representation $\Gamma_{A_{g}}$.

$$
\Gamma_{1} \otimes \Gamma_{Q} \otimes \Gamma_{2} \supset \Gamma_{A_{g}}
$$

In the linear vibronic coupling model, the totally symmetric normal modes (index $g$ ) lead to intrastate coupling, while non-totally symmetric normal modes (index $u$ ) lead to interstate coupling, if their symmetry is compatible with Equation 2 (in the point group of interest). $(\mathrm{mCP})_{2}$ is $C_{i^{-}}$ symmetric (see below), hence, only $a_{g}$ and $a_{u}$ vibrations exist. The symmetries of the two excited electronic states of interest are $A_{g}$ and $A_{u}$, thus all modes are relevant for the coupling matrix. A 
double-well structure of the lower electronic state along the respective normal mode coordinate $Q_{u}$ arises if the interstate coupling constants are sufficiently large. The totally symmetric modes (tuning modes) affect the energy separation between the coupled electronic states. ${ }^{23}$ The multimode linear vibronic coupling Hamiltonian comprising two electronic states is defined as: ${ }^{23}$

$$
\mathbf{W}^{(1)}\left(\mathbf{Q}_{\mathbf{g}}, \mathbf{Q}_{\mathbf{u}}\right)=\sum_{i, j} V_{0}\left(Q_{g i}, Q_{u j}\right) \mathbf{1}+\left(\begin{array}{cc}
E_{1}+\sum_{i} \kappa_{i}^{(1)} Q_{g i} & \sum_{j} \lambda_{j} Q_{u j} \\
\sum_{j} \lambda_{j} Q_{u j} & E_{2}+\sum_{i} \kappa_{i}^{(2)} Q_{g i}
\end{array}\right)
$$

where the ground state potential is given as $V_{0}\left(Q_{g i}, Q_{u j}\right)=\frac{\hbar \omega_{g i}}{2} Q_{g i}^{2}+\frac{\hbar \omega_{u j}}{2} Q_{u j}^{2} . E_{1,2}$ are the electronic excitation energies of the respective state, $\kappa$ is the intrastate vibronic coupling constant and $\lambda$ the interstate vibronic coupling constant. To include quadratic coupling terms, the Hamiltonian is extended. For reasons of simplicity, it is shown for a two-state/two-mode system including one tuning mode $Q_{g}$ and one coupling mode $Q_{u}$ :

$$
\begin{aligned}
\mathbf{W}^{(2)}\left(Q_{g}, Q_{u}\right)= & V_{0}\left(Q_{g}, Q_{u}\right) \mathbf{1}+ \\
& \left(\begin{array}{cc}
E_{1}+\kappa^{(1)} Q_{g}+\frac{1}{2} \gamma_{g}^{(1)} Q_{g}^{2}+\frac{1}{2} \gamma_{u}^{(1)} Q_{u}^{2} & \lambda Q_{u}+\frac{1}{2} \mu_{g u} Q_{g} Q_{u} \\
\lambda Q_{u}+\frac{1}{2} \mu_{g u} Q_{g} Q_{u} & E_{2}+\kappa^{(2)} Q_{g}+\frac{1}{2} \gamma_{g}^{(2)} Q_{g}^{2}+\frac{1}{2} \gamma_{u}^{(2)} Q_{u}^{2}
\end{array}\right)
\end{aligned}
$$

The quadratic coupling constant $\gamma$ accounts for frequency changes upon excitation. The mixed quadratic coupling $\mu_{g u}$ needs to be considered when analyzing multidimensional cuts through the potential energy surfaces (PESs). Here, we are only considering one-dimensional cuts and $\mu_{g u}$ is put to zero. Quite generally, all 2nd-order coupling terms off-diagonal in the vibrational modes ("Dushinsky mixing") are suppressed in the Hamiltonian. This is motivated by ample positive experience with the LVC model Hamiltonian which is already extended by the present approach (namely, including diagonal second-order coupling terms).

The adiabatic PES along the normal mode coordinates $Q_{g}$ and $Q_{u}$ are obtained by diagonaliza- 
tion of the diabatic coupling matrix $\mathbf{W}$ and result in the following expressions: ${ }^{23}$

$$
\begin{aligned}
V_{1,2}\left(Q_{g}\right)= & \frac{\hbar \omega_{g}}{2} Q_{g}^{2}+\frac{\gamma_{g}^{(1,2)}}{2} Q_{g}^{2}+\kappa^{(1,2)} Q_{g}+E_{1,2} \\
V_{1,2}\left(Q_{u}\right)= & \frac{\hbar \omega_{u}}{2} Q_{u}^{2}+\frac{E_{1}+E_{2}}{2}+\frac{\gamma_{u}^{(1)}+\gamma_{u}^{(2)}}{4} Q_{u}^{2} \\
& \pm \sqrt{\left(\frac{E_{2}-E_{1}}{2}+\frac{\gamma_{u}^{(2)}-\gamma_{u}^{(1)}}{4} Q_{u}^{2}\right)^{2}+\left(\lambda Q_{u}\right)^{2}}
\end{aligned}
$$

From these PESs, the intrastate coupling constant $\kappa$ and the quadratic coupling constant $\gamma$ can be derived as follows:

$$
\kappa^{(i)}=\left.\frac{\partial V_{i}(Q)}{\partial Q_{g}}\right|_{Q=0} \quad(i=1,2) \quad \gamma_{g}^{(i)}=\left.\frac{\partial^{2} V_{i}(Q)}{\partial Q_{g}^{2}}\right|_{Q=0} \quad\left(i=1,2 ; \text { for } \omega_{g}=0\right)
$$

The interstate coupling constant $\lambda$ and the corresponding quadratic coupling constants $\gamma_{u}$ are obtained from the sum and difference of the adiabatic PES, see below.

\subsection{Dynamical Calculations}

The $S_{0} \rightarrow S_{1} / S_{2}$ vibronic spectra are simulated via wave-packet propagation with the multiconfigurational time-dependent Hartree (MCTDH) method. ${ }^{28-30}$ The spectral intensity distribution $P(E)$ is obtained by Fourier transformation of the autocorrelation function $C(t) .{ }^{29}$

$$
P(E) \propto \int e^{i E t} C(t) d t, \quad \text { with } C(t)=\langle\Psi(0) \mid \Psi(t)\rangle
$$

$C(t)$ is a measure for the probability that the wave function at a time $t$ is equal to the initial wave function. MCTDH uses a multiconfigurational wave function, which is the weighted sum over Hartree products of optimized time-dependent basis functions $\phi$, called single-particle functions, 
for every degree of freedom $f$.

$$
\Psi\left(Q_{1}, \ldots, Q_{f}, t\right)=\sum_{j_{1}=1}^{n_{1}} \cdots \sum_{j_{f}=1}^{n_{f}} A_{j_{1} \cdots j_{f}}(t) \phi_{j_{1}}^{(1)}\left(Q_{1}, t\right) \cdots \phi_{j_{f}}^{(f)}\left(Q_{f}, t\right)
$$

Equations of motion for the expansion coefficients $A_{j}$ and the single-particle functions are derived through the Dirac-Frenkel variational principle.

\section{Methods}

\subsection{Computational Methods}

The mCP dimer was optimized with the approximate coupled-cluster singles and doubles method (CC2), using the resolution-of-identity (RI) and its spin-component scaled variant (SCS-CC2), ${ }^{31-33}$ and the aug-cc-pVDZ and aug-cc-pVTZ basis sets. Four minimum-energy structures were found, which are discussed below. Vertical excitation energies to the $S_{1}$ and $S_{2}$ state were calculated at the minima using the SCS-CC2 method. The spin-component-scaled variant of the CC2 method was applied because it has recently been found to yield highly reliable results for the valence excitations of aromatic molecules. ${ }^{34}$

To calculate the vibronic lines in the $S_{0} \rightarrow S_{1} / S_{2}$ spectrum, the $(\mathrm{mCP})_{2}$ ground state was optimized at the SCS-CC2/aVTZ level and normal-mode calculations were performed on this structure. One-dimensional cuts through the PESs were obtained by calculating the vertical SCS-CC2/aVTZ excitation energies at geometries that were displaced by $Q= \pm 0.5, \pm 1, \pm 2, \pm 3$ along the $S_{0}$ state normal-mode eigenvectors. All calculations were carried out using the Turbomole V6.3 program package. ${ }^{35,36}$ For the mCP monomer, the PES needed to determine the Huang-Rhys factors to evaluate the quenching factor $\Gamma$ were calculated in the same manner as for the dimer, but with the SCS-CC2/cc-pVTZ method. 


\subsection{Experimental Setup}

The $\mathrm{mCP}$ dimers are produced in a pulsed supersonic expansion of $\mathrm{mCP}$ (Aldrich, 99\%) heated to $80{ }^{\circ} \mathrm{C}$, seeded in $\mathrm{Ne}$ carrier gas at $p_{0}=1.2$ bar and expanded through a $20 \mathrm{~Hz}$ repetition rate pulsed nozzle (0.4 mm diameter). Mass-selective two-color resonant two-photon ionization (2C-R2PI) spectra were recorded by crossing the skimmed molecular beam with temporarily and spatially overlapping excitation and ionization laser beams in the source chamber of a time-of-flight mass spectrometer. For excitation, the frequency doubled output of a Nd:YAG-pumped Radiant Dyes NarrowScan dye laser (Sulforhodamine B in EtOH) with a bandwidth of $0.04 \mathrm{~cm}^{-1}$ was used. All spectra were vacuum-corrected. For the ionization step we used an Ekspla NT342B UV optical parametric oscillator/optical parametric amplifier (OPO/OPA) operated at $245 \mathrm{~nm}(1 \mathrm{~mJ} / \mathrm{pulse})$.

The fluorescence emission spectra were measured by crossing the unskimmed molecular beam with the UV excitation laser beam, fixed at the $\mathrm{mCP} 0_{0}^{0}$ band, about $4 \mathrm{~mm}$ downstream of the nozzle. The fluorescence emission was collected using a spherical mirror and quartz optics, dispersed in a SOPRA UHRS $1.5 \mathrm{~m}$ monochromator in second order and detected by a Peltier-cooled Hamamatsu R928 photomultiplier.

The ${ }^{13} \mathrm{C}$-isotopomer spectra were measured using the natural ${ }^{13} \mathrm{C}$ abundance of $15.5 \%$ for $(\mathrm{mCP})_{2}$. Deuterated mCP was obtained by dissolving $\mathrm{mCP}$ in $\mathrm{CD}_{3} \mathrm{OD}(99.8$ atom-\% D), refluxing for 3 hours, and evaporating the perdeuteromethanol, leading to $\sim 75 \%$ deuteration of the hydroxy group. The spectra of $(\mathrm{mCP}-h)(\mathrm{mCP}-d)$ were recorded using mixtures of mCP- $h$ and $\mathrm{mCP}-d$.

\section{Results}

\subsection{Equilibrium Geometries and Vibrational Analysis}

Figure 2 shows the three lowest-energy $(\mathrm{mCP})_{2}$ structures, as optimized at the SCS-CC2/augcc-pVTZ level. The CC2 and SCS-CC2 calculated relative energies are listed in Table 1. A $\pi$ stacked geometry was found at the CC2 and SCS-CC2/aug-cc-pVDZ levels, but is predicted to 
lie $270-470 \mathrm{~cm}^{-1}$ higher; its structure is shown in Figure S1 (Supporting Information). The Cartesian coordinates of the four structures in Table 1 are given in Tables S1 to S4.

While both CC2 and SCS-CC2 predict that the Z-shaped $C_{i}$-symmetric and the $C_{2 h}$-symmetric planar structure are degenerate when using the aug-cc-pVDZ basis set, the SCS-CC2/aug-cc-pVTZ calculation yields minima differing by $0.1 \mathrm{~cm}^{-1}$. With CC2/aVTZ the $C_{2 h}$ structure is an index-1 saddle point $5.5 \mathrm{~cm}^{-1}$ above the $C_{i}$ global minimum. All calculations predict a third "V-shaped" and $C_{2}$-symmetric structure $3-11 \mathrm{~cm}^{-1}$ above the global minimum. Zehnacker and co-workers optimized $(\mathrm{mCP})_{2}$ at the B3LYP/6-31G** level, which also yielded a $C_{i}$-symmetric structure. ${ }^{22}$

In $C_{i}$ symmetry all calculations predict the planes of the $\mathrm{mCP}$ subunits to be displaced along the $z$-axis, see Figure 2. The calculated displacement distance is $\Delta z= \pm 0.12 \AA$ with CC2 and $\pm 0.03 \AA$ with SCS-CC2, using the aug-cc-pVTZ basis set. Figure 2 also shows the low-frequency largeamplitude intermolecular vibrations that interconvert these structures. The $C_{2 h}$ and two symmetryequivalent $C_{i}$ minima are connected by the "stagger" vibration $\delta$. The V-shaped $C_{2}$ structure is interconverted to its enantiomer via the planar $C_{2 h}$ structure by the $\beta$ "bending" vibration. Given the tiny energy differences between the $C_{2 h}$ and $C_{i}$ minima, the barrier along the $\delta$ vibrational coordinate is probably so small that the lowest vibrational level is delocalized over all three minima. Below, we will consider the SCS-CC2/aVTZ results only.

\section{2 $S_{0} \rightarrow S_{1} / S_{2}$ Electronic Transitions}

An important difference between $(\mathrm{mCP})_{2}$ and the previously investigated H-bonded dimers ${ }^{9-18}$ is that in $(\mathrm{mCP})_{2}$ the $S_{0} \rightarrow S_{1}$ transition is ${ }^{1} A_{g} \rightarrow{ }^{1} B_{u}$ and electric-dipole allowed, while the $S_{0} \rightarrow S_{2}$ transition is ${ }^{1} A_{g} \rightarrow{ }^{1} A_{g}$ and dipole-forbidden. In the previously investigated dimers, the angle between the transition dipole moments (TDM) of the monomer moieties and the intermonomer vector $R_{A B}$ is larger than the "magic angle" $\theta_{m}=54.7^{\circ}$ for dipole $\leftrightarrow$ dipole interactions, which is given by $\cos ^{2}\left(\theta_{m}\right)=1 / 3$. The calculated TDM angles are $\theta=85^{\circ}$ in (2-pyridone) ${ }_{2},{ }^{13} 88^{\circ}$ in (benzoic acid $)_{2},{ }^{13} 59^{\circ}$ in (ortho-cyanophenol $)_{2},{ }^{17} 77^{\circ}$ in (2-aminopyridine $)_{2},{ }^{17}$ and $63^{\circ}$ in (benzonitrile) $)_{2} .{ }^{15}$ For $(\mathrm{mCP})_{2}$ however, both the CC2 and SCS-CC2 calculations predict that $\theta=11^{\circ}$, i.e., that the 
TDMs are nearly collinear, as shown in Figure 1.

The relative intensities of the $S_{0} \rightarrow S_{1}$ and $S_{0} \rightarrow S_{2}$ transitions depend on the $\theta$, as schematically depicted in Figure 3(a,b). As $\theta$ decreases, the TDM orientation changes from parallel/antiparallel, Figure 3(a), to collinear/anti-collinear in Figure 3(b). As shown to the left of Figure 3(a,b), the transition-dipole $\leftrightarrow$ transition-dipole interaction splits the $S_{1}$ and $S_{2}$ states by the energy $V_{d d}$ :

$$
V_{d d}=\frac{\left|\vec{\mu}_{A}\right|\left|\vec{\mu}_{B}\right|}{4 \pi \varepsilon_{0} R_{A B}^{3}} \cdot\left(2 \cos ^{2} \theta+\sin ^{2} \theta \cdot \cos \phi\right)
$$

where $\phi$ is zero if the two molecules are coplanar.

The calculated vertical excitation energies are given in Table 2. All calculations agree that the $S_{0} \rightarrow S_{2}$ transition is forbidden in $C_{i}$ and $C_{2 h}$ symmetries, while it is weakly allowed in $C_{2}$. The CC2 and SCS-CC2 calculated vertical excitation energies of the $C_{i}, C_{2 h}$ and $C_{2}$ isomers change only by a few $\mathrm{cm}^{-1}$ between different method/basis set combination, see Table 2, and the purely electronic $S_{1} / S_{2}$ splittings are between 173 and $201 \mathrm{~cm}^{-1}$.

\subsection{Dispersed Fluorescence Spectrum}

The dispersed fluorescence $(\mathrm{DF})$ spectrum of $(\mathrm{mCP})_{2}$ was measured by exciting at the $S_{0} \rightarrow S_{1} 0_{0}^{0}$ band $\left(33255 \mathrm{~cm}^{-1}\right)$ and is shown in Figure 4. Overall, the DF spectrum agrees well with that of Zehnacker and co-workers and many vibrational assignments are the same as those proposed in reference 22. However, we have changed the assignments of the $\delta^{\prime \prime}, \beta^{\prime \prime}, \sigma^{\prime \prime}$ and $\chi^{\prime \prime}$ intermolecular vibrations from those in reference 22 so that the agreement with the SCS-CC2/aVTZ harmonic frequencies is better. The frequencies and band assignments are listed in Table 3, together with the calculated $S_{0}$ state and experimental $S_{1}$ state frequencies.

\subsection{Two-Color Resonant Two Photon Ionization Spectrum}

The two-color resonant two-photon ionization spectrum of $(\mathrm{mCP})_{2}$ was recorded in the $33200-$ $33850 \mathrm{~cm}^{-1}$ region and is shown in Figure 5. The $S_{0} \rightarrow S_{1}$ electronic origin is at $33255 \mathrm{~cm}^{-1}$. Both 
the position of the origin and the vibronic band positions agree within $\pm 1 \mathrm{~cm}^{-1}$ with those reported in ref. 22. The vibronic bands were assigned to based on the dispersed fluorescence spectrum (see Section 4.3)and on the calculated SCS-CC2/aVTZ frequencies. We have assigned the lowest-lying $\delta^{\prime}$ and $\beta^{\prime}$ as well as the $\chi^{\prime}$ and $\sigma^{\prime}$ vibrations oppositely to those in ref. 22 , all other assignments are the same, and are listed in Table 3.

The fact that the $\delta^{\prime \prime}$ vibrational fundamental, which transforms as $a_{g}$ in $C_{i}$ is discernible in the $\mathrm{DF}$ and is clear in the R2PI spectrum strongly suggests that the $S_{0}$ state structure is effectively $C_{i}$ symmetric. As discussed in section 4.1 , the $v^{\prime \prime}=1$ and higher levels of the $\delta^{\prime \prime}$ vibration may be delocalized over the $C_{2 h}$ and both $C_{i}$ structures. However, since the $\beta$ vibration is non-totally symmetric $\left(a_{u}\right)$ in $C_{i}$ symmetry it should not appear as a fundamental, as is indeed the case for the DF spectrum, where $\beta_{0}^{1}$ does not appear but $\beta_{0}^{2}$ is observed. In the R2PI spectrum, we observe a weak band at $0_{0}^{0}+15.4 \mathrm{~cm}^{-1}$, which we tentatively ascribe to $\beta_{0}^{1}$. We have considered several possibilities why this fundamental does appear:

(1) Existence of several different $S_{0}$ state forms, as is predicted by the calculations. In this case, the R2PI spectra that originate from the ground state $v=0$ levels of the different forms should be separable by UV/UV hole-burning spectroscopy. However, UV/UV holeburning spectra measured when burning at the origin band, shown as Figure S2 (Supplemental Information) did not reveal the presence of two (or more) separable v"=0 species.

(2) Excited-state $(\mathrm{mCP})_{2}$ is effectively $C_{2}$-symmetric. While this would make the $\beta^{\prime}$ vibration totally symmetric $(a), \delta^{\prime}$ then becomes non-totally-symmetric $(b)$, leading to a problem in explaining the appearance of the $\delta^{\prime}$ fundamental.

(3) Zehnacker and co-workers proposed a loss of symmetry upon electronic excitation which would lead to the appearance of the additional fundamentals. ${ }^{22}$ Due to the excitonic coupling, the $S_{1}$ state adiabatic PES has a double-minimum shape along one or more antisymmetric vibrations. ${ }^{24}$ While each of the adiabatic $S_{1}$ state minima have lower local symmetry $\left(C_{1}\right)$, the molecular symmetry (MS) group of the coupled $S_{1} / S_{2}$ states remains $C_{i}$, and the symmetry-restriction rules still apply. The question of the (a)symmetry of the coupled $S_{1} / S_{2}$ excited states is discussed in detail 
below.

(4) For intermolecular complexes of aromatics with noble-gas atoms, Felker and co-workers have shown that vibronic transitions which are nominally forbidden as fundamentals by rigidmolecule selection rules may nevertheless gain significant intensity if they involve intermolecular vibrations in which the aromatic moiety - which carries the electronic transition-dipole moment (TDM) - librates with sufficiently large amplitude, which renders such transitions vibronically active. ${ }^{37} \mathrm{In}(\mathrm{mCP})_{2}$ the out-of-plane intermolecular bending vibration $\beta^{\prime}$ librationally modulates the electronic TDMs of both monomers, and this may render the $\beta^{\prime}$ fundamental active in the spectrum.

Considering the very small energy differences between the $S_{0}$ state minima discussed in Section 4 , we expect the potential energy surface along the $\delta$ and $\beta$ normal coordinates to be very flat. It is therefore probable that the lowest-frequency vibrations are delocalized over several ground state structures. The $\delta$-mode interconnects the $C_{i}$-minima via the $C_{2 h}$-structure, while the $\beta$-mode interconnects the two $\mathrm{V}$-shape $C_{2}$ minima via the $C_{2 h}$-structure. The $\delta$ mode is $a_{g}$ in $C_{i}$ and $\beta$ is $a$ in $C_{2}$ symmetry, respectively, but not in $C_{2 h}$. On the other hand, their first overtone is fully allowed in all symmetries. This might explain the intensities of the measured spectrum: Both fundamental transitions are weak, while the corresponding overtones are much more intense.

\section{Discussion}

\subsection{Excitonic Splitting and Isotope Effects}

Isotopic substitution by even a single ${ }^{13} \mathrm{C}$ or a $\mathrm{D}$ atom breaks the inversion symmetry of $(\mathrm{mCP})_{2}$ strongly enough to render also the $S_{0} \rightarrow S_{2}$ transition allowed. The 2C-R2PI spectra of both ${ }^{12} \mathrm{C} /{ }^{13} \mathrm{C}$-substituted and H/D-substituted dimers were measured, in order to break the dimer symmetry in two ways, allowing to measure two $S_{1} / S_{2}$ splittings. Figure 6(a) compares the origin region of all- ${ }^{12} \mathrm{C}-(\mathrm{mCP})_{2}$ to that of ${ }^{13} \mathrm{C}-(\mathrm{mCP})_{2}$ in Figure $6(\mathrm{~b})$. The ${ }^{13} \mathrm{C}-(\mathrm{mCP})_{2}$ spectrum shows an additional weak band at $33264 \mathrm{~cm}^{-1}$, about $8 \mathrm{~cm}^{-1}$ above the intense $S_{0} \rightarrow S_{1} 0_{0}^{0}$ transition at 
$33256 \mathrm{~cm}^{-1}$. The former is assigned to the $S_{0} \rightarrow S_{2} 0_{0}^{0}$ transition, giving the excitonic splitting $\Delta_{o b s,{ }^{13} \mathrm{C}}=8.0 \pm 0.4 \mathrm{~cm}^{-1}$.

${ }^{12} \mathrm{C} /{ }^{13} \mathrm{C}$ isotopic contribution: The changes in the zero-point vibrational energy (ZPVE) upon isotopic substitution lead to an ${ }^{12} \mathrm{C} /{ }^{13} \mathrm{C}$ isotopic shift of the $0_{0}^{0}$ transition $\Delta_{i s o}$ which contributes to the splitting. In the dimers (benzoic acid) ${ }_{2}{ }^{14}$ and (benzonitrile) $)_{2},{ }^{15}$ the analogous isotopic shifts were $\Delta_{o b s{ }^{13} \mathrm{C}}=3.3 \mathrm{~cm}^{-1}$ and $\Delta_{o b s{ }^{13} \mathrm{C}}=3.9 \mathrm{~cm}^{-1}$, respectively. These small shifts may be treated by second-order degenerate perturbation theory, as $\Delta_{o b s}{ }^{13} C=\sqrt{\Delta_{\text {exc }}^{2}+\Delta_{\text {iso, }{ }^{13} C}^{2}}$. The $\Delta_{i s o,{ }^{13} C}$ contribution was determined by measuring the monomer $2 \mathrm{C}$-R2PI spectra of $\mathrm{mCP}$ and ${ }^{13} \mathrm{C}-\mathrm{mCP}$. Figure 7 shows that the $S_{0} \rightarrow S_{1}$ origin of ${ }^{13} \mathrm{C}-\mathrm{mCP}$ is shifted by $+3.3 \pm 0.4 \mathrm{~cm}^{-1}$ relative to that of $\mathrm{mCP}$. Thus, the purely excitonic contribution $\Delta_{e x c}$ to the splitting between the $S_{1}$ and $S_{2}$ origin bands is $\Delta_{\text {exc }}=\sqrt{\Delta_{o b s,{ }^{13} C}^{2}-\Delta_{\text {iso },{ }^{13} C}^{2}}=7.3 \pm 0.5 \mathrm{~cm}^{-1}$.

$H / D$ isotope shift: Figure 6(c) shows the origin region of (mCP- $h)(\mathrm{mCP}-d)$. The intense $S_{0} \rightarrow S_{1}$ $0_{0}^{0}$ transition lies at $33260 \mathrm{~cm}^{-1}$, here the weaker $S_{0} \rightarrow S_{2} 0_{0}^{0}$ band appears at $33270 \mathrm{~cm}^{-1}$. The latter transition is more intense compared to of ${ }^{13} \mathrm{C}-(\mathrm{mCP})_{2}$, which indicates a stronger symmetry breaking by H/D exchange as compared to ${ }^{12} \mathrm{C} /{ }^{13} \mathrm{C}$ substitution, and also a stronger exciton localization, see below. The observed $S_{1} / S_{2}$ splitting is $\Delta_{o b s, D}=10 \mathrm{~cm}^{-1}$, or $2 \mathrm{~cm}^{-1}$ larger than for the ${ }^{13} \mathrm{C}$-isotopomer. $\mathrm{H} / \mathrm{D}$ substitution changes the ZPVE than ${ }^{12} \mathrm{C} /{ }^{13} \mathrm{C}$ exchange, because the relative change of mass is much larger. The R2PI spectra of $(\mathrm{mCP}-d)(\mathrm{mCP}-h)$ and $(\mathrm{mCP}-d)_{2}$ are compared to that of $(\mathrm{mCP})_{2}$ in Figure 8 . The R2PI spectrum of non-deuterated $(\mathrm{mCP})_{2}$ shown in Figure 8(a) shows the same vibronic structure as that of the doubly O-H/O-D deuterated homodimer $(\mathrm{mCP}-d)_{2}$, Figure $8(\mathrm{c})$. However, in the $(\mathrm{mCP}-d)(\mathrm{mCP}-h)$ heterodimer, the inversion symmetry is broken, rendering the R2PI spectrum much more complex as both the $S_{0} \rightarrow S_{1}$ and $S_{0} \rightarrow S_{2}$ transitions are allowed, giving rise to more vibronic bands, see Figure 8(b). The excitonic splitting $\Delta_{\text {exc }}=7.3 \mathrm{~cm}^{-1}$ determined above allows to extract $\Delta_{i s o, D}=6.8 \mathrm{~cm}^{-1}$ from the observed splitting $\Delta_{o b s, D}$ as $\Delta_{i s o, D}=\sqrt{\Delta_{o b s, D}^{2}-\Delta_{e x c}^{2}}=10 \mathrm{~cm}^{-1}$ as $6.8 \mathrm{~cm}^{-1}$. 
Vibronic quenching: To relate the observed excitonic splitting of $\Delta_{\text {exc }}=7.3 \mathrm{~cm}^{-1}$ to the calculated electronic Davydov-splitting $\Delta_{e l}$ (e.g., $179 \mathrm{~cm}^{-1}$ with the SCS-CC2/aVTZ method), the latter must be reduced or "quenched" by the dimensionless vibronic quenching factor $\Gamma_{\text {vibron }}$, using Förster's perturbation theory ansatz: ${ }^{13}$

$$
\Delta_{\text {vibron }}=\Delta_{e l} \cdot \Gamma_{\text {vibron }}
$$

The vibronic quenching factor, $\Gamma_{\text {vibron }}=\prod_{i} \exp \left(-S_{i}\right)$ is the product of the individual vibrational quenching factors $\gamma_{i}=\exp \left(-S_{i}\right) \leq 1$, where $i$ ranges over all totally-symmetric vibrational modes of the monomer and $S_{i}$ is the $i$-th dimensionless vibrational displacement between the $S_{0}$ and $S_{1}$ state along the vibrational coordinate $Q_{i}$ (Huang-Rhys factor). $S_{i}$ is a measure for the intensity of the corresponding vibronic line and can be obtained (1) experimentally, from the intensities of totally-symmetric vibronic band fundamentals in the dispersed fluorescence spectrum of the mCP monomer, giving $\Gamma_{\text {vibron }}^{\text {exp }}{ }^{15}$ (2) computationally from the $\kappa_{i}$ coupling parameters of the monomer $S_{0} \leftrightarrow S_{1}$ transition, as described in section 5.2, giving $\Gamma_{\text {vibron }}^{\text {calc }},{ }^{15}$ (3) following a different computational path, $\Gamma_{\text {vibron }}$ can be calculated via the effective-mode approximation, giving $\Gamma_{v i b r o n}^{e f f}{ }^{24}$

Table 4 lists the mode-specific quenching factors $\gamma_{i}$ of the $\mathrm{mCP}$ monomer and the total vibronic quenching factors $\Gamma_{\text {vibron }}^{\text {exp }}$ and $\Gamma_{\text {vibron }}^{c a l c}$. After quenching the calculated Davydov splitting $\Delta_{e l}=$ $179 \mathrm{~cm}^{-1}$ with $\Gamma_{\text {vibron }}^{\text {exp }}$ (column 4 of Table 4) one obtains $\Delta_{\text {vibron }}^{\text {exp }}=7.7 \mathrm{~cm}^{-1}$, which is in excellent agreement with the experimentally observed splitting $\Delta_{e x c}=7.3 \mathrm{~cm}^{-1}$. When quenching $\Delta_{e l}$ with $\Gamma_{\text {vibron }}^{c a l c}$, we obtain $\Delta_{\text {vibron }}^{\text {calc }}=24.2 \mathrm{~cm}^{-1}$; the same value is obtained upon quenching with $\Gamma_{v i b r o n}^{e f f}$. The latter two values are more than three times larger than experimentally observed. The reason for this can be seen in columns 2 and 3 of Table 4, which shows that many of the Huang-Rhys factors $\gamma_{i}$ calculated for the $S_{\leftrightarrow} S_{1}$ transition of $\mathrm{mCP}$ are lower than the experimental ones. 


\subsection{Determination of the $(\mathrm{mCP})_{2}$ Coupling Parameters}

The coupling constants necessary for the MCTDH simulation were obtained by taking the SCS$\mathrm{CC} 2 / \mathrm{aVTZ} C_{i}$-symmetric ground state equilibrium geometry as reference geometry in the vibronic coupling formalism and subsequently distorting along every normal mode. The inter- and intrastate coupling constants $(\lambda$ and $\kappa)$ were determined from least squares fits of the analytical expressions for the adiabatic surfaces (Eqs. 5 and 6, omitting $\omega_{g / u}$ ) to the ab initio calculated excitation energies. This procedure has been successfully employed for the dimers of 2-pyridone and of orthocyanophenol. ${ }^{16,18}$ The coupling parameters for the $a_{g}$ and $a_{u}$ normal modes with a frequency up to $600 \mathrm{~cm}^{-1}$ are listed in Tables 5 and 6, respectively. Modes marked by an asterisk are considered in the LVC and QVC simulations. The corresponding normal-mode eigenvectors are shown in Figures 9 and 10. The criterion for including a mode in the LVC and QVC simulations is that its frequency is smaller than $600 \mathrm{~cm}^{-1}$ and that its Huang-Rhys factor is $S_{i}>0.02$.

Figure 11 shows the fits to the excitation energies along the selected $a_{g}$ modes. The quadratic coupling constant $\gamma$ is a measure of frequency change: A negative/positive curvature of the excitation energies indicates a frequency lowering/increase upon electronic excitation. ${ }^{16}$ For the $a_{u}$ vibrations, the coupling constants are calculated from fits to the sum and the difference of the adiabatic potential energy curves along the antisymmetric normal coordinates $Q_{u}$, shown in Figure 12. The curvature of the difference of the adiabatic PES is a measure of the linear coupling constant $\lambda$ : The stronger the curvature, the larger $\lambda$.

\subsection{Vibronic Spectrum Simulation}

The simulation of the excited state vibronic spectrum was performed with the MCTDH program package $^{38}$ using the linear (LVC) and quadratic (QVC) vibronic coupling models. Both simulations are shown in Figure 13(b) and (c) along the experimental spectrum, Figure 13(a), in the spectral region up to $600 \mathrm{~cm}^{-1}$. All spectra are set to a common origin. The simulations include seven totally-symmetric $\left(a_{g}\right)$ and five $a_{u}$ modes. The corresponding coupling constants are collected in Tables 5 and 6 . We account for the higher-frequency modes by introducing a prequenched 
energy gap of $59.0 \mathrm{~cm}^{-1}$ between the two electronic states, which is determined by treating the high-frequency coupling modes within Förster's perturbation theory approach. For details on the prequenching, see ref. 18; the prequenching concept has successfully been applied to the dimers of ortho-cyanophenol and 2-pyridone. ${ }^{16,18}$ In all cases, the ground state potentials are taken to be harmonic with frequencies $\omega_{g / u}$. The LVC simulation only contains the linear coupling parameters $\kappa$ and $\lambda$, while the QVC calculation additionally includes the quadratic coupling parameters $\gamma$. For mode $v_{1}$ we introduce an exception in the QVC simulation. In this case the combination of the harmonic ground state with the quadratic coupling terms would lead to an unphysical behavior of the PES (unbound from below). To stay in line with our model approach we therefore neglect the quadratic coupling terms for mode $v_{1}$. The initial wave function is chosen as a product of harmonic oscillator functions. The line assignments for the LVC and QVC spectra are collected in Table 7 and are based on the nodal structures of the respective vibronic densities.

In the LVC simulation shown in Figure 13(b), the calculated frequencies are close to the experimentally observed ones shown in Figure 13(a), especially below $400 \mathrm{~cm}^{-1}$, see also Table 3 . The intensities of the stagger $(\delta)$ and in-plane nitrogen-bend $(\delta \mathrm{CC}-\mathrm{N})$ are well reproduced. The intensities of the $\sigma$ transitions are underestimated by the simulation compared to the experiment. The same applies to $\chi$. It may be due to the different ground state structures present that the transitions of $\sigma$ and $\chi$ are larger in the experimental spectrum, as these vibrations are totally-symmetric for all geometries. Also, the band intensities of R2PI spectra are not as reliable as for example the intensities of DF spectra, because they are sensitive to temperature and saturation effects. Nonetheless, the structure of the simulated spectrum shows a similarity to the observed spectrum, although fewer bands could be reproduced, especially in the low-energy region. In the high-energy region, $6 a_{0}^{1}$ shows combinations with $\delta_{0}^{1}, \sigma_{0}^{1}$ and $\chi_{0}^{1}$, which agrees well with experiment. The intensity of $6 a_{0}^{1}$ is overestimated. In general, the frequencies in the spectrum above $400 \mathrm{~cm}^{-1}$ are not well reproduced by the LVC simulation and the intensities are overestimated.

The QVC simulation in Figure 13(c) shows intensities closer to experiment in the low-energy region compared to the LVC spectrum, especially for the $\sigma$ bands. The $6 a_{0}^{1}$ intensity is overes- 
timated, while the intensity of $\delta \mathrm{CC}-\mathrm{N}_{0}^{1}$ and $6 b_{0}^{1}$ are very reasonable. Also, the frequencies have changed markedly. For the higher energy region of the spectrum $\left(\geq 400 \mathrm{~cm}^{-1}\right)$, the frequencies of the QVC simulation are closer to the R2PI spectrum than the LVC frequencies. However, for the low-energy region the opposite is the case: Most frequencies are somewhat too small in the QVC simulation, although the deviation to experiment is reduced compared to the LVC results.

While the frequencies for the totally symmetric modes in the calculated spectra well match the harmonic ground state frequencies, for the coupling modes $\omega, \delta \mathrm{C}-\mathrm{OH}_{a s}$ and $6 \mathrm{a}_{a s}$ the frequencies for excitation of one quantum are larger in the LVC model than the ground state frequency. In the QVC scheme, this increase is compensated by the negative quadratic coupling constants. Regarding symmetry selection rules, the excitation of odd quanta in the coupling modes should not be observed. However, they appear because, due to the coupling of the electronic $S_{1}$ and $S_{2}$ states, the vibronic wave functions have components associated with both electronic states. As demonstrated earlier, this results in non-adiabatic effects and may lead to complicated spectral features. ${ }^{16,18}$

The overall shape of the spectrum is reproduced although certain bands of the out-plane vibrations are missing in the simulation because the Huang-Rhys factor is too small. As discussed previously, the use of Cartesian displacement coordinates can be problematic for the low-frequency out-of-plane modes. ${ }^{16}$ Table 7 gives an overview over the observed and simulated frequencies for the LVC and QVC model.

The extremely small Huang-Rhys factors of out-of-plane modes (see Tables 5 and 6) indicate that the coupling parameters may not be of good quality. The displacement around the equilibrium geometry $(Q=0)$ is performed along the Cartesian normal-mode eigenvectors of every vibration. Especially at large displacements this may lead to incorrect bond length changes. This problem can be overcome by employing internal coordinates, rather than the Cartesian normal-mode eigenvectors. This has the advantage that no bond length changes are mixed into angular deformations, but is beyond the scope of this work. 


\subsection{Localization of the Excitation}

The localization of the excitation in the different species is determined in the same way as for (benzoic acid $)_{2}$ and (benzonitrile $)_{2} \cdot{ }^{14,15}$ For both homodimers $(\mathrm{mCP}-h)_{2}$ and $(\mathrm{mCP}-d)_{2}$, only the $S_{0} \rightarrow S_{1}$ transition is observed, see Figure 8. Therefore, the excitation is fully delocalized over both monomers. Breaking the inversion symmetry leads to a partial localization. ${ }^{14,15}$ Degenerate perturbation theory allows to determine the localization of the wave functions by means of the mixing angle $\alpha$, which is defined as $\tan (2 \alpha)=\left|\Delta_{\text {exc }}\right| /\left|E_{A^{*} B}-E_{A B^{*}}\right| ;{ }^{19}$ for details, see refs. 14 and 15.

First we consider the ${ }^{13} \mathrm{C}$-isotopomer, where $\alpha=21.1^{\circ}$. Therefore, $\Psi^{+}$is $86 \%$ localized on monomer $A\left({ }^{12} \mathrm{C}-\mathrm{mCP}\right)$ and only $14 \%$ on monomer $B\left({ }^{13} \mathrm{C}-\mathrm{mCP}\right)$, and vice versa for $\Psi^{-}$. For $(\mathrm{mCP}-h)(\mathrm{mCP}-d) \alpha=18.1^{\circ}$, leading to $\Psi^{+}$being $90 \%$ localized on monomer $A(\mathrm{mCP}-h)$ and $10 \%$ localized on monomer $B(\mathrm{mCP}-d)$, and again vice versa for $\Psi^{-}$. These ratios can be compared to experimental observations via the dimer oscillator strengths $f_{\text {dim }}^{ \pm}$, see refs. 14 and 15 . For ${ }^{13} \mathrm{C}-(\mathrm{mCP})_{2}$, a calculated ratio of $f_{\text {dim }}^{+}: f_{\text {dim }}^{-}=1: 0.19$ results. The integrated intensities of the experimental $S_{0} \rightarrow S_{1}$ and $S_{0} \rightarrow S_{2}$ origins shown in Figure 6 are in good agreement, being 1 : 0.10. This indicates that the determined isotopic and excitonic contributions are reliable. From the determined splitting in $(\mathrm{mCP}-h)(\mathrm{mCP}-d)$, the calculated intensity ratio is $f_{\text {dim }}^{+}: f_{d i m}^{-}=1: 0.26$; the experiment yields $1: 0.30$, in excellent agreement. Consequently, the ${ }^{13} \mathrm{C}$-substitution leads to a smaller localization of the excitation compared to partial deuteration of mCP. Nevertheless, in both heterodimers the monomers are distinguishable in the excited state. The excitation can oscillate back and forth between the monomers upon excitation with a large enough bandwidth to cover both origins. 19

For the homodimers $(\mathrm{mCP})_{2}$ and $(\mathrm{mCP}-d)_{2}$ the angle is $\alpha=\pi / 4$; the semiclassical exciton transfer rate is $k_{A B}=4\left|V_{A B}\right| / h=2\left|\Delta_{e x c}\right| \cdot c,{ }^{19}$ resulting in a transfer rate of $k_{A B}=4.4 \cdot 10^{11} \mathrm{~s}^{-1}$, which is equivalent to an exciton hopping time of $t_{\text {exc }}=\left(k_{A B}\right)^{-1}=2.3$ ps. This is much faster compared to the benzoic acid dimer $(17.7 \mathrm{ps})^{14}$ and the benzonitrile dimer $(8.0 \mathrm{ps}) .{ }^{15}$ A possible reason is the much smaller intermolecular distance: $R_{A B}=5.34 \AA$ for $(\mathrm{mCP})_{2}$, while in $(\mathrm{BZA})_{2}$ 
and $(\mathrm{BN})_{2}$ the monomers are $7.14 \AA$ and $6.53 \AA$ apart, respectively. ${ }^{14}$

For the heterodimers, the excitation is partially localized, therefore the transfer rate should decrease and the hopping time increase compared to the electronically delocalized homodimers. The transfer rate of ${ }^{13} \mathrm{C}-(\mathrm{mCP})_{2}$ is determined to be $k_{A B}=1.6 \cdot 10^{11} \mathrm{~s}^{-1}$, corresponding to $t_{\text {exc }}=$ $6.3 \mathrm{ps}$. The increase in hopping time is very small compared to $(\mathrm{mCP})_{2}$, agreeing with a partial localization. For $(\mathrm{mCP}-h)(\mathrm{mCP}-d)$, the transfer rate is $k_{A B}=7.1 \cdot 10^{10} \mathrm{~s}^{-1}$. The decrease in $k_{A B}$ is a bit larger compared to the ${ }^{13} \mathrm{C}-(\mathrm{mCP})_{2}$. The hopping time is therefore a little longer, $t_{\text {exc }}=14$ ps. Thus, the excitation is slightly more localized in $(\mathrm{mCP}-h)(\mathrm{mCP}-d)$ compared to ${ }^{13} \mathrm{C}-(\mathrm{mCP})_{2}$.

\subsection{Asymmetry of the First Excited State}

Due to the appearance of the out-of-plane fundamental $\beta_{0}^{1}$, Seurre et al. suggested a symmetry loss upon excitation breaking the inversion center. ${ }^{22}$ Based on this they concluded a localization of the excitation on the monomers. The $S_{1}$ state geometry optimization of $(\mathrm{mCP})_{2}$ shows indeed an asymmetric deformation. Both rings expand upon excitation, but the $\mathrm{C}-\mathrm{C}$ bond lengths in monomer $A$ increase by $28-54 \mathrm{pm}$, while they increase only by $12-14 \mathrm{pm}$ in monomer $B$. This leads to a $C_{1}$ symmetry. Table 8 details the bond lengths and structure parameters of the $S_{0}$ and $S_{1}$ states.

For symmetry reasons, there is a second dimer structure, in which the deformations on $A$ and $B$ are exchanged. This structure corresponds to the symmetry-equivalent and degenerate minimum of the double-well type $S_{1}$ adiabatic potential of $(\mathrm{mCP})_{2}$. Both the $S_{1}$ and $S_{2}$ states of the dimer are delocalized over both minima, therefore, the geometry change does not imply localization of the excitation in an asymmetric structure. This is illustrated by the adiabatic PESs of the first and second excited state along the antisymmetric effective mode coordinate $Q_{-}^{\text {eff }}$ in Figure 14 . The aspect of localization in case of isotopic substitution is treated above in detail. 


\section{Conclusions}

The $S_{1} / S_{2}$ excitonic splitting of the $C_{i}$-symmetric dimer (meta-cyanophenol $)_{2}$ is not directly measurable by optical spectroscopy, because its $S_{0} \rightarrow S_{1}\left(A_{g} \rightarrow A_{u}\right)$ transition is electric-dipole allowed, while the $S_{0} \rightarrow S_{2}\left(A_{g} \rightarrow A_{g}\right)$ transition is dipole-forbidden. A single ${ }^{12} \mathrm{C} /{ }^{13} \mathrm{C}$ or $\mathrm{H} / \mathrm{D}$ substitution breaks the inversion symmetry of $(\mathrm{mCP})_{2}$ sufficiently to render the $S_{0} \rightarrow S_{2}$ transition somewhat allowed. We have measured mass-selected two-color resonant two-photon ionization spectra of the $S_{0} \rightarrow S_{1}$ and $S_{0} \rightarrow S_{2}$ electronic origins of ${ }^{12} \mathrm{C} /{ }^{13} \mathrm{C}$ or H/D substituted dimers of mCP. Combining the information of the ${ }^{13} \mathrm{C}-(\mathrm{mCP})_{2}$ and $(\mathrm{mCP}-h)(\mathrm{mCP}-d)$ heterodimer spectra allows to experimentally determine the excitonic splitting of $(\mathrm{mCP})_{2}$ as $\Delta_{e x c}=7.3 \mathrm{~cm}^{-1}$.

$\mathrm{CC} 2$ and SCS-CC2 calculations predict three close-lying ground-state structures of $(\mathrm{mCP})_{2}$ : The lowest is near-planar but slightly Z-shaped ( $C_{i}$-symmetric, two enantiomers), the secondlowest structure is $0-5 \mathrm{~cm}^{-1}$ higher and is planar and $C_{2 h}$-symmetric. The third structure is $3-11 \mathrm{~cm}^{-1}$ higher and is V-shaped $\left(C_{2}\right.$-symmetric, two enantiomers). CC2 and SCS-CC2/aVDZ calculations predict a $\pi$-stacked dimer geometry, which is $270-470 \mathrm{~cm}^{-1}$ higher; this structure is not a minimum at the SCS-CC2/aVTZ level.

The isotope-dependent shifts in the $S_{0} \rightarrow S_{1} / S_{2}$ origin bands of the ${ }^{13} \mathrm{C}-(\mathrm{mCP})_{2}$ and (mCP$h)(\mathrm{mCP}-d)$ heterodimers arise from the changes of the vibrational zero-point energy between the electronic ground and excited states. The isotope shift that arises from a single ${ }^{12} \mathrm{C} /{ }^{13} \mathrm{C}$ substitution was determined as $\Delta_{i s o}{ }_{13}^{13} \mathrm{C}=3.3 \mathrm{~cm}^{-1}$; we do not observe any dependence on the position of the ${ }^{13} \mathrm{C}$ atom within the dimer, although there must be small effects. The isotope effect that arises from $\mathrm{H} / \mathrm{D}$ exchange in the phenolic $\mathrm{OH}$ group is somewhat larger, being $\Delta_{i s o, D}=6.8 \mathrm{~cm}^{-1}$. This leads to a larger splitting between the vibrationless levels of the $S_{1}$ and $S_{2}$ states.

The purely electronic (vertical) excitonic splitting calculated with the SCS-CC2 method and the aug-cc-pVTZ basis set is $\Delta_{e l}=179 \mathrm{~cm}^{-1}$. The large difference between this value and the experimental $\Delta_{\text {exc }}=7.3 \mathrm{~cm}^{-1}$ reflects the importance of vibronic coupling, which reduces or quenches the purely electronic splitting. The vibronic quenching factor $\Gamma_{\text {vibron }}=\Pi_{i} \gamma_{i}$ that is experimentally determined from the $\mathrm{mCP}$ dispersed fluorescence spectrum, $\Gamma_{\text {vibron }}^{\exp }=0.043$, yields 
$\Delta_{\text {vibron }}^{\text {exp }}=\Delta_{e l} \cdot \Gamma_{\text {vibron }}^{\text {exp }}=7.7 \mathrm{~cm}^{-1}$, in very good agreement with the experiment. The quenching factor $\Gamma_{\text {vibron }}^{c a l c}$ derived from the mCP monomer calculated $S_{0} \rightarrow S_{1}$ spectrum is three times larger, resulting in a $\Delta_{\text {vibron }}^{\text {calc }}=24.2 \mathrm{~cm}^{-1}$.

Our conclusion that the excitonic excitations are delocalized is in disagreement with the interpretation of Seurre et al., ${ }^{22}$ but not with their experimental observations. The excitonic coupling between $S_{1}$ and $S_{2}$ gives rise to two asymmetric $S_{1}$ state minimum-energy geometries, corresponding to a potential with two symmetry-equivalent minima along the antisymmetric effective mode coordinate $Q_{-}^{e f f}$. While the rigid-molecule symmetry at these minima is reduced from $C_{i}$ to $C_{1}$, the molecular symmetry (MS) group of the $S_{1}$ state including tunneling remains $C_{i}(\mathrm{M}){ }^{15-18}$

We interpret the appearance of the fundamental of the low-frequency out-of-plane mode $\beta^{\prime}$ in the $S_{0} \rightarrow S_{1}$ spectra as deriving from the strongly librational character of the $\beta$ vibration, which modulates the electronic TDM strongly enough to induce the appearance of fundamental transitions in this nominally antisymmetric mode by a Herzberg-Teller-like mechanism. ${ }^{37}$

The simulation of the vibronic spectrum with MCTDH agrees fundamentally with the structure of the in-plane modes observed experimentally. Issues arose for the description of out-of-plane modes, as the predicted Huang-Rhys parameters were far too small to cause these modes to appear in the spectrum. Apparently, the geometry displacement along the normal-mode eigenvectors is not the best description and internal coordinates would be necessary for a complete description and simulation of the out-of-plane modes. Nevertheless, the simulations agrees with the observed spectrum and differences of linear and quadratic vibronic coupling have been illustrated.

\section{Acknowledgments}

This work was supported by the Schweiz. Nationalfonds (project nos. 200020-152816 and 132540) and the Deutsche Forschungsgemeinschaft (project no. AZ KO945/17-1).

Supporting Information Available: Figure $S 1$ with the $C_{2}$-symmetric, stacked structure of the meta-cyanophenol dimer (not observed experimentally). Figure S2 with UV/UV-holeburned spec- 
trum, Tables S1 to S4 with Cartesian coordinates of the SCS-CC2/aug-cc-pVTZ optimized four structures in Table 1.

\section{References}

(1) Cheng, Y. C.; Fleming, G. Dynamics of Light Harvesting in Photosynthesis. Annu. Rev. Phys. Chem. 2009, 60, 241-262.

(2) Novoderezhkin, V. I.; van Grondelle, R. Physical Origins and Models of Energy Transfer in Photosynthetic Light-Harvesting. Phys. Chem. Chem. Phys. 2010, 12, 7352-7365.

(3) Strümpfer, J.; Schulten, K. Excited State Dynamics in Photosynthetic Reaction Center and Light Harvesting Complex 1. J. Chem. Phys. 2012, 137, 065101-1-7.

(4) Fassioli, F.; Dinshaw, R.; Arpin, P. C.; Scholes, G. D. Photosynthetic Light Harvesting: Excitons and Coherence. J. Roy. Soc. Interface 2014, 11, 20130901-1-22.

(5) Scholes, G. D.; Smith, C. Detecting and Measuring Exciton Delocalization in Photosynthetic Light Harvesting. J. Chem. Phys. 2014, 140, 110901-1-8.

(6) Spano, F. C. Excitons in Conjugated Oligomer Aggregates, Films, and Crystals. Annu. Rev. Phys. Chem. 2006, 57, 217-243.

(7) Markovitsi, D.; Onidas, D.; Gustavsson, T.; Talbot, F.; Lazzarotto, E. Collective Behavior of Franck-Condon Excited States and Energy Transfer in DNA Double Helices. J. Am. Chem. Soc. 2005, 127, 17130-17131.

(8) Middleton, C. T.; de La Harpe, K.; Su, C.; Law, Y. K.; Crespo-Hernandez, C. E.; Kohler, B. DNA Excited-State Dynamics: From Single Bases to the Double Helix. Annu. Rev. Phys. Chem. 2009, 60, 217-239. 
(9) Müller, A.; Talbot, F.; Leutwyler, S. $S_{1} / S_{2}$ Exciton Splitting in the (2-Pyridone) 2 Dimer. $J$. Chem. Phys. 2002, 116, 2836-2847.

(10) Ottiger, P.; Leutwyler, S.; Köppel, H. $S_{1} / S_{2}$ Excitonic Splittings and Vibronic Couplings in the Excited-State of the Jet-Cooled 2-Aminopyridine Dimer. J. Chem. Phys. 2009, 131, 204308-1-11.

(11) Ottiger, P.; Leutwyler, S. Excitonic Splittings in Jet-Cooled Molecular Dimers. CHIMIA 2011, 65, 228-230.

(12) Heid, C. G.; Ottiger, P.; Leist, R.; Leutwyler, S. The $S_{1} / S_{2}$ Exciton Interaction in 2-Pyridone-6-Methyl-2-Pyridone: Davydov Splitting, Vibronic Coupling and Vibronic Quenching Effects. J. Chem. Phys. 2011, 135, 154311-1-15.

(13) Ottiger, P.; Leutwyler, S.; Köppel, H. Vibrational Quenching of Excitonic Splittings in HBonded Molecular Dimers: The Electronic Davydov Splittings Cannot Match Experiment. J. Chem. Phys. 2012, 136, 174308-1-13.

(14) Ottiger, P.; Leutwyler, S. Excitonic Splitting and Coherent Electronic Energy Transfer in the Gas-Phase Benzoic Acid Dimer. J. Chem. Phys. 2012, 137, 204303.

(15) Balmer, F. A.; Ottiger, P.; Leutwyler, S. Excitonic Splitting, Delocalization, and Vibronic Quenching in the Benzonitrile Dimer. J. Phys. Chem. A 2014, 118, 11253-11261.

(16) Kopec, S.; Ottiger, P.; Leutwyler, S.; Köppel, H. Analysis of the $S_{2} \leftarrow S_{0}$ Vibronic Spectrum of the ortho-Cyanophenol Dimer Using a Multimode Vibronic Coupling Approach. J. Chem. Phys. 2015, 142, 084308-1-16.

(17) Ottiger, P.; Köppel, H.; Leutwyler, S. Excitonic Splittings in Molecular Dimers: Why Static Ab Initio Calculations Cannot Match Them. Chem. Sci 2015, 6, 6059-6068.

(18) Kopec, S.; Köppel, H. Theoretical Analysis of the $S_{0} \rightarrow S_{2}$ Vibronic Spectrum of the 2Pyridone Dimer. J. Chem. Phys. 2016, 144, 024314-1-13. 
(19) Förster, T. In Modern Quantum Chemistry; Sinanoglu, O., Ed.; Academic Press, New York, 1965; Chapter III. B, p 93.

(20) Fulton, R. L.; Gouterman, M. Vibronic Coupling. I. Mathematical Treatment for Two Electronic States. J. Chem. Phys. 1961, 35, 1059-1071.

(21) Fulton, R. L.; Gouterman, M. Vibronic Coupling. II. Spectra of Dimers. J. Chem. Phys. 1964, $41,2280-2286$.

(22) Seurre, N.; Barbu-Debus, K. L.; Lahmani, F.; Zehnacker-Rentien, A.; Sepiol, J. Electronic and Vibrational Spectroscopy of Jet-Cooled m-Cyanophenol and its Dimer: Laser-Induced Fluorescence and Fluorescence-Dip IR Spectra in the $S_{0}$ and $S_{1}$ states. Chem. Phys. 2003, $295,21-33$.

(23) Köppel, H.; Domke, W.; Cederbaum, L. S. Multimode Molecular Dynamics Beyond the Born-Oppenheimer Approximation. Adv. Chem. Phys. 1984, 57, 59-245.

(24) Kopec, S.; Ottiger, P.; Leutwyler, S.; Köppel, H. Vibrational Quenching of Excitonic Splittings in H-Bonded Molecular Dimers: Adiabatic Description and Effective Mode Approximation. J. Chem. Phys. 2012, 137, 184312-1-10.

(25) Nebgen, B.; Emmert, F. L.; Slipchenko, L. V. Vibronic Coupling in Asymmetric Bichromophores: Theory and Application to Diphenylmethane. J. Chem. Phys. 2012, 137, 084112$1-12$.

(26) Nebgen, B.; Slipchenko, L. V. Vibronic Coupling in Asymmetric Bichromophores: Theory and Application to Diphenylmethane-d5. J. Chem. Phys. 2014, 141, 134119-1-9.

(27) Pillsbury, N. R.; Kidwell, N. M.; Nebgen, B.; Slipchenko, L. V.; Douglass, K. O.; Cable, J. R.; Plusquellic, D. F.; Zwier, T. S. Vibronic Coupling in Asymmetric Bichromophores: Experimental Investigation of Diphenylmethane-d $\mathrm{d}_{5}$. J.Chem. Phys. 2014, 141, 064316-1-9. 
(28) Meyer, H.-D.; Manthe, U.; Cederbaum, L. S. The Multi-Configurational Time-Dependent Hartree Approach. Chem. Phys. Lett. 1990, 165, 73-78.

(29) Meyer, H.-D.; Gatti, F.; Worth, G. A. Multidimensional Quantum Dynamics: MCTDH Theory and Application; Wiley-VCH, 2009.

(30) Beck, M. H.; Jäckle, A.; Worth, G. A.; Meyer, H.-D. The Multiconfigurational TimeDependent Hartree (MCTDH) Method: A Highly Efficient Algorithm For Propagating Wavepackets. Phys. Rep. 2000, 324, 1-105.

(31) Hättig, C.; Weigend, F. CC2 Excitation Energy Calculations on Large Molecules using the Resolution of the Identity Approximation. J. Chem. Phys. 2000, 113, 5154-5161.

(32) Hättig, C. Geometry Optimizations with the Coupled-Cluster Model CC2 using the Resolution of the Identity Approximation. J. Chem. Phys. 2003, 118, 7751-7761.

(33) Hellweg, A.; Grün, S. A.; Hättig, C. Benchmarking the Performance of Spin-component Scaled CC2 in Ground and Electronically Excited States. Phys. Chem. Chem. Phys. 2008, $10,4119-4127$.

(34) Winter, N.; Graf, N. K.; Leutwyler, S.; Hättig, C. Benchmarks for 0-0 Transitions of Aromatic Organic Molecules: DFT/B3LYP, ADC(2), CC2, SOS-CC2 and SCS-CC2 Compared to High-Resolution Gas-Phase Data. Phys. Chem. Chem. Phys. 2013, 15, 6623-6630.

(35) TURBOMOLE V6.3 2011; a development of Universität Karlsruhe (TH) and Forschungszentrum Karlsruhe GmbH, 1989-2007, TURBOMOLE GmbH; available from http://www.turbomole.com.

(36) The thresholds for SCF and one-electron density convergence were set to $10^{-9}$ a.u. and $10^{-8}$ a.u., respectively. The convergence thresholds for all structure optimizations were set to $10^{-8}$ a.u. for the energy change, $6 \cdot 10^{-6}$ a.u. for the maximum displacement element, 
$10^{-6}$ a.u. for the maximum gradient element, $4 \cdot 10^{-6}$ a.u. for the RMS displacement and $10^{-6}$ a.u. for the RMS gradient.

(37) Maxton, P. M.; Schaeffer, M. W.; Ohline, S. M.; Kim, W.; Venturo, V.; Felker, P. M. The Raman and Vibronic Activity of Intermolecular Vibrations in Aromatic-Containing Complexes and Clusters. J. Chem. Phys. 1994, 101, 8391-8408.

(38) Worth, G. A.; Beck, M. H.; Jäckle, A.; Meyer, H.-D. The MCTDH Package, Version 8.4. 2007; University of Heidelberg, Heidelberg, Germany. http://mctdh.uni-hd.de. 
Table 1: Calculated Relative Energies $\left(\mathrm{in}^{-1}\right)$ of the Four Lowest-Energy $(m \text {-Cyanophenol })_{2}$ Structures, see Figure 2.

\begin{tabular}{lcccc}
\hline \hline & Z-shaped & Planar & V-shaped & $\pi$-Stacked \\
& $C_{i}$ & $C_{2 h}$ & $C_{2}$ & $C_{2}$ \\
\hline CC2/aug-cc-pVDZ & 0.0 & 0.0 & 11.3 & 474.4 \\
CC2/aug-cc-pVTZ & 0.0 & $5.5^{\mathrm{b}}$ & 7.5 & 315.9 \\
SCS-CC2/aug-cc-pVDZ & 0.0 & 0.0 & 11.4 & 272.6 \\
SCS-CC2/aug-cc-pVTZ & 0.0 & 0.1 & 3.1 & - \\
\hline \hline
\end{tabular}

a shown in Figure S1 (Supplemental Information).

$\mathrm{b}$ index-1 saddle point 
Table 2: Calculated Vertical Excitation Energies and Davydov-splitting Energies $\Delta_{e l}\left(\mathrm{in} \mathrm{cm}^{-1}\right)$ of the H-bonded ( $m$-Cyanophenol $)_{2}$ Structures, see Figure 2.

\begin{tabular}{|c|c|c|c|c|c|c|c|c|c|}
\hline \multirow[b]{2}{*}{ Z-shaped $\left(C_{i}\right)$} & \multirow[b]{2}{*}{ Irred. } & \multicolumn{2}{|c|}{ "CC2/aVDZ } & \multicolumn{2}{|c|}{ CC2/aVTZ } & \multicolumn{2}{|c|}{ "SCS-CC2/aVDZ } & \multicolumn{2}{|c|}{ "SCS-CC2/aVTZ } \\
\hline & & $\mathrm{E}_{e x c}$ & $f_{e l}$ & $\mathrm{E}_{e x c}$ & $f_{e l}$ & $\mathrm{E}_{e x c}$ & $f_{e l}$ & $\mathrm{E}_{\text {exc }}$ & $f_{e l}$ \\
\hline$S_{1}$ & $A_{u}$ & 35369 & 0.157 & 35849 & 0.160 & 35412 & 0.123 & 35935 & 0.134 \\
\hline$S_{2}$ & $A_{g}$ & 35563 & 0.000 & 36045 & 0.000 & 35585 & 0.000 & 36114 & 0.000 \\
\hline$\Delta_{e l}$ & & 194 & & 196 & & 173 & & 179 & \\
\hline \multicolumn{10}{|l|}{ Planar $\left(C_{2 h}\right)$} \\
\hline$S_{1}$ & $B_{u}$ & 35369 & 0.157 & 35845 & 0.160 & 35411 & 0.123 & 35935 & 0.134 \\
\hline$S_{2}$ & $A_{g}$ & 35562 & 0.000 & 36046 & 0.000 & 35585 & 0.000 & 36115 & 0.000 \\
\hline$\Delta_{e l}$ & & 193 & & 201 & & 174 & & 180 & \\
\hline \multicolumn{10}{|l|}{ V-shaped $\left(C_{2}\right)$} \\
\hline$S_{1}$ & $B$ & 35370 & 0.157 & 35187 & 0.152 & 35412 & 0.123 & 35936 & 0.134 \\
\hline$S_{2}$ & $A$ & 35562 & $4 \cdot 10^{-4}$ & 35379 & $4 \cdot 10^{-4}$ & 35585 & $3 \cdot 10^{-4} 3 \mathrm{E}-04$ & 36115 & $3 \cdot 10^{-4}$ \\
\hline$\Delta_{e l}$ & & 194 & & 192 & & 173 & & 179 & \\
\hline
\end{tabular}


Table 3: Experimental $S_{0}$ and $S_{1}$ Frequencies (in $\left.\mathrm{cm}^{-1}\right)$ of ( $m$-Cyanophenol $)_{2}$ and SCS-CC2/augcc-pVTZ $S_{0}$ Frequencies and Comparison to Assignments of Ref. 22.

\begin{tabular}{|c|c|c|c|c|c|c|}
\hline Vibration $S_{1}$ & 2C-R2PI & Ref. 22(S $\left(S_{1}\right)$ & Vibration $S_{0}$ & Fluorescence & SCS-CC2/aVTZ & Ref. $22\left(S_{0}\right)$ \\
\hline$\delta_{0}^{1}$ & 12.8 & 30 & $\delta_{1}^{0}$ & 15 & 11 & 34 \\
\hline$\beta_{0}^{1}$ & 15.4 & 12 & $\beta_{1}^{0}$ & $(17.5)^{\mathrm{a}}$ & 13 & 11 \\
\hline$\delta_{0}^{2}$ & 27.3 & & $\beta_{2}^{0}$ & 35 & & \\
\hline$\beta_{0}^{2}$ & 30.4 & & $\sigma_{1}^{0}$ & 75 & 79 & 104 \\
\hline$\beta_{0}^{4}$ & 60.8 & & $\chi_{1}^{0}$ & 103 & 109 & 75 \\
\hline$\theta_{0}^{2}$ & 70.6 & & $\delta_{1}^{0} \chi_{1}^{0}$ & 119 & & \\
\hline$\sigma_{0}^{1}$ & 84.5 & 75 & $\sigma_{2}^{0}$ & 148 & & \\
\hline$\sigma_{0}^{1} \delta_{0}^{1}$ & 97.3 & & $\delta \mathrm{CC}-\mathrm{N}_{1}^{0}$ & 168 & 171 & 165 \\
\hline$\theta_{0}^{2} \delta_{0}^{2}$ & 99.3 & & & & & \\
\hline$\sigma_{0}^{1} \delta_{0}^{2}$ & 111.2 & & & & & \\
\hline$\chi_{0}^{1}$ & 116.9 & 84 & & & & \\
\hline$\chi_{0}^{1} \delta_{0}^{1}$ & 127.6 & & & & & \\
\hline$\sigma_{0}^{2}$ & 167.1 & & & & & \\
\hline$\delta \mathrm{CC}-\mathrm{N}_{0}^{1}$ & 176.7 & & & & & \\
\hline$\sigma_{0}^{2} \delta_{0}^{1}$ & 180.0 & & & & & \\
\hline$\sigma_{0}^{2} \delta_{0}^{2}$ & 193.8 & & & & & \\
\hline$\chi_{0}^{2}$ & 225.8 & & & & & \\
\hline$\sigma_{0}^{3}$ & 248.4 & & & & & \\
\hline$\sigma_{0}^{3} \delta_{0}^{1}$ & 263.4 & & & & & \\
\hline$\sigma_{0}^{3} \delta_{0}^{2}$ & 275.0 & & & & & \\
\hline$\delta \mathrm{C}-\mathrm{OH}_{0}^{1}$ & 394.9 & & & & & \\
\hline$\delta \mathrm{C}-\mathrm{OH}_{0}^{1} \delta_{0}^{1}$ & 407.6 & & & & & \\
\hline $6 a_{0}^{1}$ & 413.5 & & & & & \\
\hline $6 a_{0}^{1} \delta_{0}^{1}$ & 425.0 & & & & & \\
\hline $6 a_{0}^{1} \beta_{0}^{1}$ & 430.8 & & & & & \\
\hline $6 a_{0}^{1} \delta_{0}^{2}$ & 439.4 & & & & & \\
\hline$\delta \mathrm{C}-\mathrm{OH}_{0}^{1} \sigma_{0}^{1}$ & 478.5 & & & & & \\
\hline $6 b_{0}^{1}$ & 496.6 & & & & & \\
\hline $6 a_{0}^{1} \sigma_{0}^{1}$ & 498.8 & & & & & \\
\hline$\delta \mathrm{C}-\mathrm{CN}_{0}^{1}$ & 523.5 & & & & & \\
\hline $6 a_{0}^{1} \chi_{0}^{1}$ & 529.8 & & & & & \\
\hline $6 a_{0}^{1} \sigma_{0}^{2}$ & 578.0 & & & & & \\
\hline $6 b_{0}^{1} \sigma_{0}^{1}$ & 582.6 & & & & & \\
\hline
\end{tabular}

Frequency from the first overtone. 
Table 4: SCS-CC2/cc-pVTZ calculated and experimental frequencies (in $\mathrm{cm}^{-1}$ ) of the totallysymmetric vibrations of $m$-cyanophenol, calculated and experimental mode-specific quenching factors $\gamma_{i}=\exp \left(-S_{i}\right)$, calculated and experimental total quenching factors $\Gamma_{\text {vibron }}^{\text {calc }}$ and $\Gamma_{\text {vibron }}^{\exp }$.

\begin{tabular}{|c|c|c|c|}
\hline \multicolumn{2}{|c|}{ SCS-CC2/cc-pVTZ } & \multicolumn{2}{|c|}{ Experimental } \\
\hline Frequency/cm ${ }^{-1}$ & $\gamma_{i, \text { calc }^{\mathrm{a}}}$ & Freq. $/ \mathrm{cm}^{-1}$ & $\gamma_{i, \exp }{ }^{\mathrm{b}}$ \\
\hline 141.8 & 0.975 & 146.5 & 0.942 \\
\hline 387.4 & 0.991 & & \\
\hline 450.9 & 0.652 & 453.2 & 0.439 \\
\hline 527.7 & 0.817 & 531.2 & 0.637 \\
\hline 558.2 & 0.975 & 564.1 & 0.960 \\
\hline 720.7 & 0.887 & 718.0 & 0.867 \\
\hline 942.5 & 0.942 & 935.7 & 0.842 \\
\hline 1003.9 & 0.660 & 1004.4 & 0.446 \\
\hline 1093.8 & 0.977 & 993.7 & 0.900 \\
\hline 1167.4 & 0.924 & & \\
\hline 1180.0 & 0.993 & & \\
\hline 1195.5 & 0.995 & & \\
\hline 1299.4 & 0.856 & 1286.5 & 0.591 \\
\hline 1339.4 & 0.931 & & \\
\hline 1436.2 & 0.993 & & \\
\hline 1466.0 & 0.950 & & \\
\hline 1517.8 & 0.997 & & \\
\hline 1629.7 & 0.999 & & \\
\hline 1634.1 & 0.988 & & \\
\hline 2106.2 & 0.904 & & \\
\hline 3201.2 & 0.950 & & \\
\hline 3214.6 & 0.989 & & \\
\hline 3231.3 & 0.993 & & \\
\hline 3239.9 & 0.982 & & \\
\hline 3793.4 & 0.893 & & \\
\hline$\Gamma_{\text {calc }}$ & 0.135 & $\Gamma_{\exp }$ & 0.0434 \\
\hline$\Delta_{\text {vibron }}^{\text {calc }}$ & $24.2 \mathrm{~cm}^{-1}$ & $\Delta_{\text {vibron }}^{\text {calc }}$ & $7.7 \mathrm{~cm}^{-1}$ \\
\hline
\end{tabular}

a from calculated coupling constants of $\mathrm{mCP}$.

${ }^{b}$ from fluorescence band intensities of mCP. 
Table 5: Vibronic coupling parameters (in $\mathrm{cm}^{-1}$ ) based on SCS-CC2/aVTZ excited state potential fits for totally symmetric $a_{g}$ modes. The mode number corresponds to the Turbomole numbering, for spectral reference the Wilson nomenclature is given in parenthesis. $\omega_{g}$ is the calculated frequency, $\kappa$ the intrastate coupling constant, $\gamma$ the quadratic coupling parameter and $S_{i}$ the HuangRhys factor.

\begin{tabular}{|c|c|c|c|c|c|}
\hline$a_{g}$-Mode & $\omega_{g}$ & State & $\kappa$ & $\gamma$ & $S_{i}$ \\
\hline \multirow[t]{2}{*}{$1^{*}(\delta)$} & 10.8 & $S_{1}$ & -4.8 & -459.4 & 0.100 \\
\hline & & $S_{2}$ & 1.0 & -463.0 & 0.004 \\
\hline \multirow[t]{2}{*}{$4^{*}(\sigma)$} & 79.2 & $S_{1}$ & 55.1 & -9.7 & 0.242 \\
\hline & & $S_{2}$ & 49.6 & -9.9 & 0.196 \\
\hline \multirow[t]{2}{*}{$6^{*}(\chi)$} & 108.5 & $S_{1}$ & -30.3 & -14.5 & 0.039 \\
\hline & & $S_{2}$ & -27.3 & -14.3 & 0.032 \\
\hline \multirow[t]{2}{*}{7} & 139.2 & $S_{1}$ & -4.5 & -63.2 & 0.001 \\
\hline & & $S_{2}$ & -4.7 & -63.2 & 0.001 \\
\hline \multirow[t]{2}{*}{$9 *(\delta \mathrm{CC}-\mathrm{N})$} & 171.4 & $S_{1}$ & 79.9 & -43.8 & 0.109 \\
\hline & & $S_{2}$ & 73.5 & -43.1 & 0.092 \\
\hline \multirow[t]{2}{*}{11} & 221.7 & $S_{1}$ & -3.5 & -42.4 & $1.2 \mathrm{E}-04$ \\
\hline & & $S_{2}$ & -3.9 & -44.9 & $1.6 \mathrm{E}-04$ \\
\hline \multirow[t]{2}{*}{13} & 381.1 & $S_{1}$ & 2.7 & -168.5 & $2.5 \mathrm{E}-05$ \\
\hline & & $S_{2}$ & 3.4 & -168.7 & $3.9 \mathrm{E}-05$ \\
\hline \multirow[t]{2}{*}{$16(\delta \mathrm{C}-\mathrm{OH})$} & 412.7 & $S_{1}$ & -21.7 & -2.6 & 0.001 \\
\hline & & $S_{2}$ & -27.3 & -3.5 & 0.002 \\
\hline \multirow[t]{2}{*}{$18 *(6 a)$} & 458.2 & $S_{1}$ & 321.1 & -34.5 & 0.246 \\
\hline & & $S_{2}$ & 319.7 & -35.7 & 0.243 \\
\hline \multirow[t]{2}{*}{19} & 460.5 & $S_{1}$ & 36.1 & -66.8 & 0.003 \\
\hline & & $S_{2}$ & 36.0 & -70.6 & 0.003 \\
\hline \multirow[t]{2}{*}{$21 *(6 b)$} & 528.4 & $S_{1}$ & 229.1 & -34.4 & 0.094 \\
\hline & & $S_{2}$ & 208.8 & -35.7 & 0.078 \\
\hline \multirow[t]{2}{*}{$23 *(\delta \mathrm{C}-\mathrm{CN})$} & 569.4 & $S_{1}$ & -136.3 & -0.3 & 0.029 \\
\hline & & $S_{2}$ & -132.3 & -1.3 & 0.027 \\
\hline
\end{tabular}


Table 6: Vibronic coupling parameters (in $\mathrm{cm}^{-1}$ ) based on SCS-CC2/aVTZ excited state potential fits for non-totally symmetric modes of $a_{u}$ symmetry. The mode number corresponds to the Turbomole numbering, for spectral reference the Wilson nomenclature is given in parenthesis. $\omega_{u}$ is the calculated frequency, $\lambda$ the interstate coupling constant, $\gamma$ the quadratic coupling parameter and $S_{i}$ the Huang-Rhys factor.

\begin{tabular}{lcrrrc}
\hline \hline$a_{u}$-Mode & $\omega_{u}$ & $\lambda$ & $\gamma_{1}$ & $\gamma_{2}$ & $S_{i}$ \\
\hline $2(\beta)$ & 13.0 & 0.03 & -377.0 & -373.6 & $1.9 \mathrm{E}-06$ \\
$3(\theta)$ & 47.2 & 0.05 & -21.7 & -23.4 & $5.2 \mathrm{E}-07$ \\
$5 *(\omega)$ & 99.5 & 35.4 & -52.6 & -38.2 & 0.063 \\
8 & 143.6 & 0.6 & -61.4 & -61.4 & $7.6 \mathrm{E}-06$ \\
$10\left(\delta \mathrm{CC}-\mathrm{N}_{a s}\right)$ & 183.8 & 12.0 & -30.2 & -30.3 & 0.002 \\
12 & 224.2 & 0.1 & -44.2 & -45.1 & $9.5 \mathrm{E}-08$ \\
14 & 382.3 & 8.3 & -170.0 & -170.0 & $2.4 \mathrm{E}-04$ \\
$15^{*}\left(\delta \mathrm{C}-\mathrm{OH}_{a s}\right)$ & 412.1 & 117.9 & -7.4 & -2.3 & 0.041 \\
$17 *\left(6 \mathrm{a}_{a s}\right)$ & 458.2 & 298.0 & -33.9 & -33.8 & 0.212 \\
20 & 465.3 & 2.1 & -69.0 & -71.9 & $1.0 \mathrm{E}-05$ \\
$22^{*}\left(6 \mathrm{~b}_{a s}\right)$ & 528.5 & 204.5 & -35.1 & -35.2 & 0.075 \\
$24 *\left(\delta \mathrm{C}-\mathrm{CN}_{a s}\right)$ & 571.8 & 113.9 & -3.7 & 6.8 & 0.020 \\
\hline \hline
\end{tabular}


Table 7: Vibrational frequencies (in $\mathrm{cm}^{-1}$ ) in the experimental 2C-R2PI spectrum and in the linear (LVC) and quadratic (QVC) MCTDH simulations.

\begin{tabular}{lccc}
\hline \hline Vibration & 2C-R2PI & LVC & QVC \\
\hline$\delta_{0}^{1}$ & 12.8 & 10.8 & 10.7 \\
$\sigma_{0}^{1}$ & 84.5 & 79.2 & 74.2 \\
$\sigma_{0}^{1} \delta_{0}^{1}$ & 97.3 & 90.0 & 85.0 \\
$\chi_{0}^{1}$ & 116.9 & 108.5 & 101.0 \\
$\omega_{0}^{1}$ & & 128.9 & 93.3 \\
$\sigma_{0}^{2}$ & 167.1 & 158.5 & 148.3 \\
$\delta \mathrm{CC}-\mathrm{N}_{0}^{1}$ & 176.7 & 171.4 & 148.0 \\
$\sigma_{0}^{1} \chi_{0}^{1}$ & & 187.8 & 175.2 \\
$\sigma_{0}^{1} \omega_{0}^{1}$ & & 208.0 & 167.5 \\
$\sigma_{0}^{1} \delta \mathrm{CC}-\mathrm{N}_{0}^{1}$ & & 250.6 & 222.1 \\
$\chi_{0}^{1} \delta \mathrm{CC}-\mathrm{N}_{0}^{1}$ & & 279.9 & 249.1 \\
$\omega_{0}^{1} \delta \mathrm{CC}-\mathrm{N}_{0}^{1}$ & & & 241.4 \\
$\delta \mathrm{C}-\mathrm{OH} \mathrm{H}_{a s}$ & & 442.3 & 435.3 \\
$6 \mathrm{a}_{0}^{1}$ & 413.5 & 458.5 & 440.8 \\
$6 \mathrm{a}_{0}^{1} \delta_{0}^{1}$ & 425.0 & 469.4 & 451.5 \\
$\left(6 \mathrm{a}_{a s}\right)_{0}^{1}$ & & 479.0 & 457.8 \\
$6 \mathrm{~b}_{0}^{1}$ & 496.6 & 528.4 & 511.1 \\
$6 \mathrm{a}_{0}^{1} \sigma_{0}^{1}$ & 498.5 & 537.8 & 514.9 \\
$\delta \mathrm{C}-\mathrm{CN} \mathrm{N}_{0}^{1}$ & 523.5 & 569.6 & 569.4 \\
\hline \hline
\end{tabular}


Table 8: Characteristic structure parameters (in $\AA$ ) of (meta-cyanophenol) $)_{2}$ in the SCS-CC2/aVTZ optimized $S_{0}$ and $S_{1}$ states. N' stands for the N atom of $B$.

\begin{tabular}{lccc}
\hline \hline Parameter & \multicolumn{2}{c}{$S_{0}\left(C_{i}\right)$} & \multicolumn{2}{c}{$S_{1}\left(C_{1}\right)$} \\
& $\mathrm{A}=\mathrm{B}$ & $\mathrm{A}$ & $\mathrm{B}$ \\
\hline$r\left(\mathrm{C}_{1}-\mathrm{C}_{2}\right)$ & 1.397 & 1.443 & 1.410 \\
$r\left(\mathrm{C}_{2}-\mathrm{C}_{3}\right)$ & 1.403 & 1.435 & 1.416 \\
$r\left(\mathrm{C}_{3}-\mathrm{C}_{4}\right)$ & 1.394 & 1.431 & 1.407 \\
$r\left(\mathrm{C}_{4}-\mathrm{C}_{5}\right)$ & 1.398 & 1.426 & 1.410 \\
$r\left(\mathrm{C}_{4}-\mathrm{C}_{5}\right)$ & 1.399 & 1.453 & 1.412 \\
$r\left(\mathrm{C}_{5}-\mathrm{C}_{6}\right)$ & 1.402 & 1.446 & 1.416 \\
$r\left(\mathrm{C}_{6}-\mathrm{C}_{7}\right)$ & 1.437 & 1.419 & 1.449 \\
$r\left(\mathrm{C}_{7}-\mathrm{N}_{8}\right)$ & 1.176 & 1.200 & 1.190 \\
$r\left(\mathrm{C}_{9}-\mathrm{O}_{10}\right)$ & 1.356 & 1.349 & 1.365 \\
$r\left(\mathrm{O}_{10}-\mathrm{H}_{11}\right)$ & 0.976 & 0.991 & 0.982 \\
$\delta\left(\mathrm{C}_{6}-\mathrm{C}_{7}-\mathrm{N}_{8}\right)$ & $174.7^{\circ}$ & $173.2^{\circ}$ & $174.6^{\circ}$ \\
$\delta\left(\mathrm{C}_{9}-\mathrm{O}_{10}-\mathrm{H}_{11}\right)$ & $110.2^{\circ}$ & $110.8^{\circ}$ & $110.2^{\circ}$ \\
$r\left(\mathrm{H}_{11} \cdots \mathrm{N}^{\circ}{ }_{8}\right)$ & 2.025 & \multicolumn{2}{c}{1.978} \\
$r\left(\mathrm{H}_{11} \cdots \mathrm{N}^{\prime}\right)$ & 2.025 & \multicolumn{2}{c}{1.914} \\
$R_{C O M}$ & 5.340 & \multicolumn{2}{c}{5.341} \\
$\Delta_{z}$ & 0.006 & \multicolumn{2}{c}{0.004} \\
\hline \hline
\end{tabular}




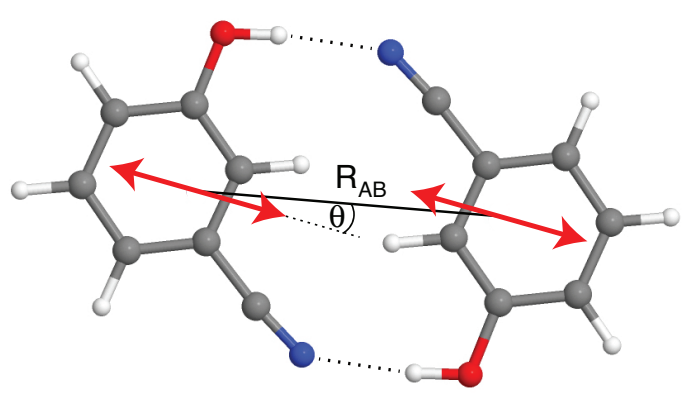

Figure 1: SCS-CC2/aug-cc-pVTZ structure and electronic transition dipole moment (TDM) directions of (meta-cyanophenol) $)_{2}$. The distance between the centers of mass is $R_{A B}=5.34 \AA$, the angle between the intermonomer distance vector and the TDMs is $\theta=11^{\circ}$. 
1

2

3

4

5

6

8

10

11

12

13

14

15

16

17

18

19

20

21

22

23

24

25

26

27

28

29

30

31

32

33

34

35

36

37

38

39

40

41

42

43

44

45

46

47

48

49

50

51

52

53

54

55

56

57

58

59

60

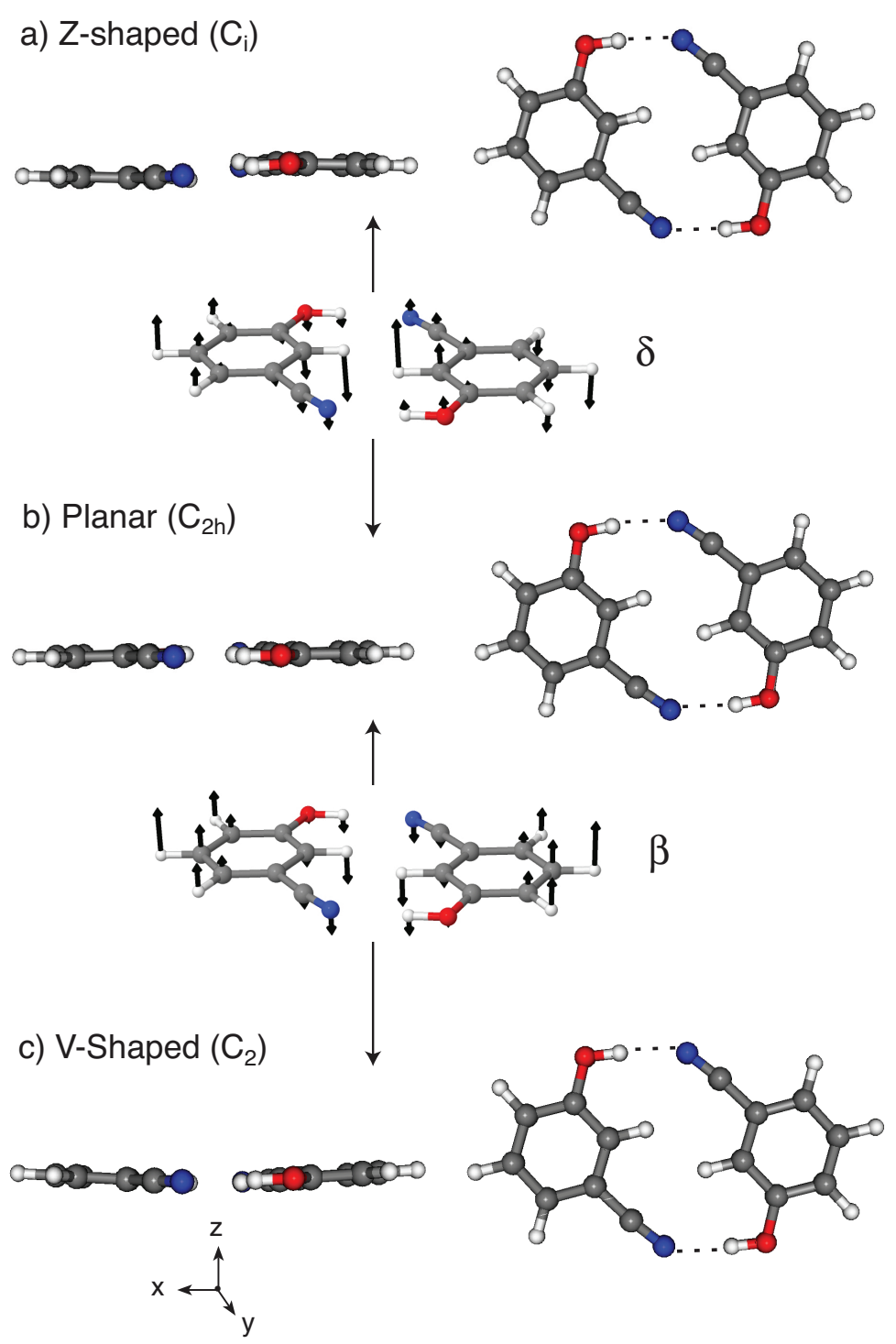

Figure 2: SCS-CC2/aug-cc-pVTZ optimized isomers of (meta-cyanophenol) $)_{2}$. Left: side views, right: top views. The intermolecular vibrations $\delta$ and $\beta$ interconvert the $C_{i}, C_{2 h}$ and $C_{2}$ structures. 


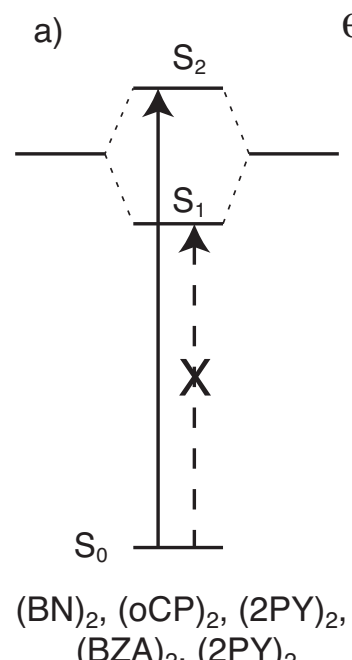

$\theta>54.7^{\circ}$

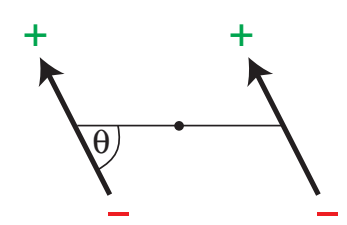

Figure 3: Electric-dipole allowed and forbidden $S_{0} \rightarrow S_{1} / S_{2}$ transitions (left) and corresponding transition dipole-moment vector directions in excitonic homodimers. (a) For $\theta>54.7^{\circ}$, (b) for $\theta<54.7^{\circ}$. Full and dashed vertical arrows symbolize allowed and forbidden electronic transitions. Examples of spectroscopically investigated homodimers are given for both cases, see the text. 


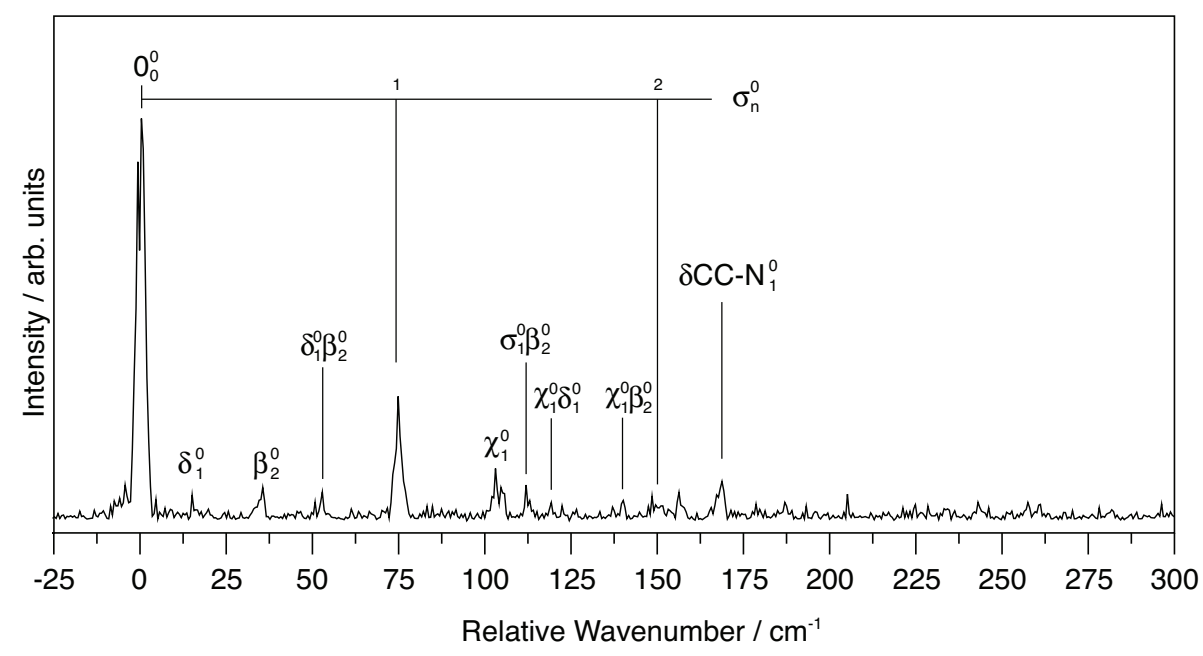

Figure 4: Dispersed fluorescence spectrum of ( $m$-cyanophenol $)_{2}$ with band assignments. The wavenumber scale is relative to the $0_{0}^{0}$ band at $33255 \mathrm{~cm}^{-1}$. 


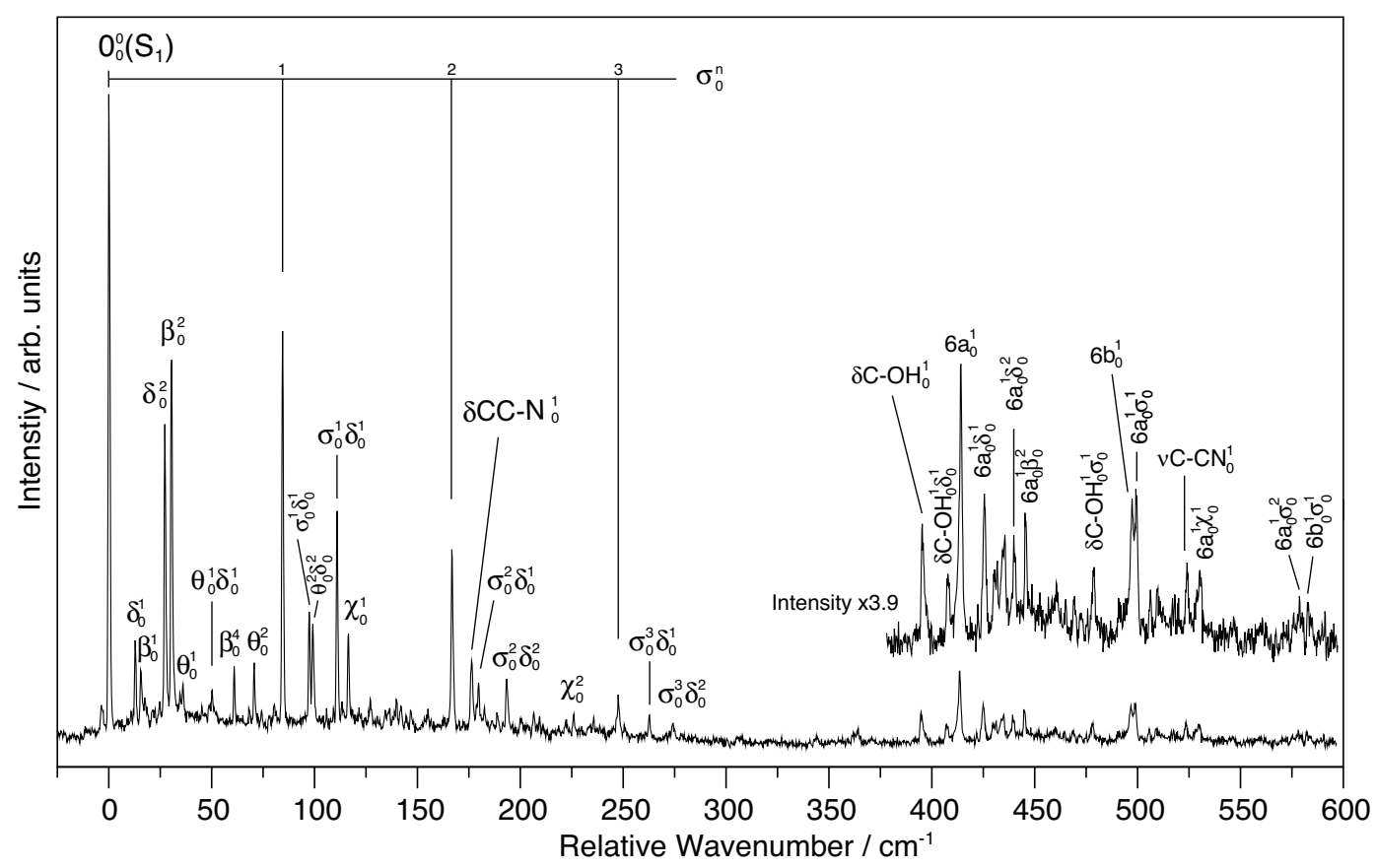

Figure 5: Two-color resonant two-photon ionization spectrum of (meta-cyanophenol) 2 with assigned vibronic transitions. The scale is relative to the $0_{0}^{0}$ band at $33255 \mathrm{~cm}^{-1}$. 


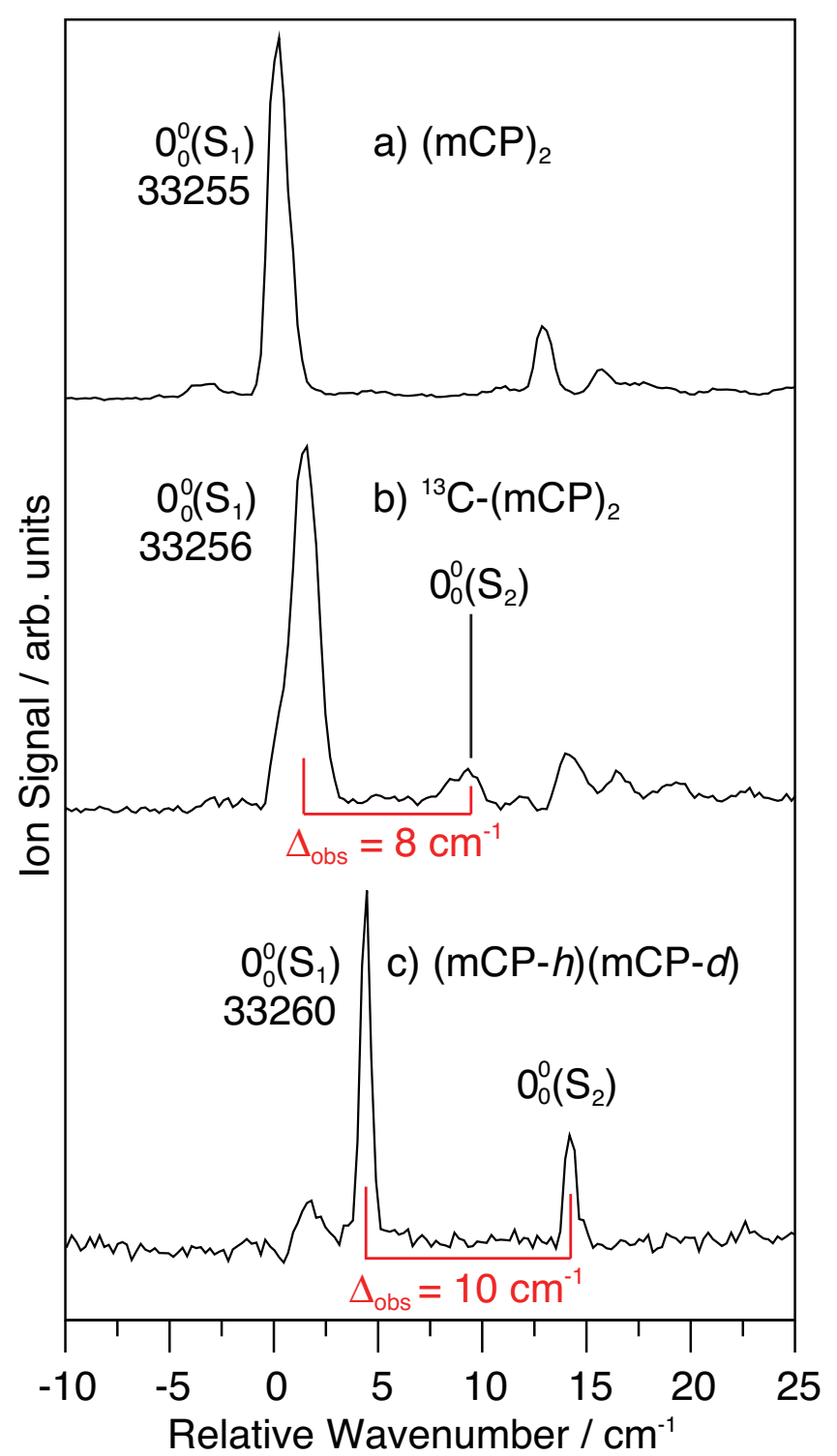

Figure 6: 2C-R2PI spectra of (a) (meta-cyanophenol) $)_{2}$, (b) ${ }^{13} \mathrm{C}$-substituted and c) H/D substituted heterodimers in the $0_{0}^{0}$ band region. The scale is relative to the $0_{0}^{0}$ band of $(\mathrm{mCP})_{2}$ at $33255 \mathrm{~cm}^{-1}$. The $0_{0}^{0}$ band of ${ }^{13} \mathrm{C}-(\mathrm{mCP})_{2}$ is at $33256 \mathrm{~cm}^{-1}$, that of $(\mathrm{mCP}-h)(\mathrm{mCP}-d)$ at $33260 \mathrm{~cm}^{-1}$. 


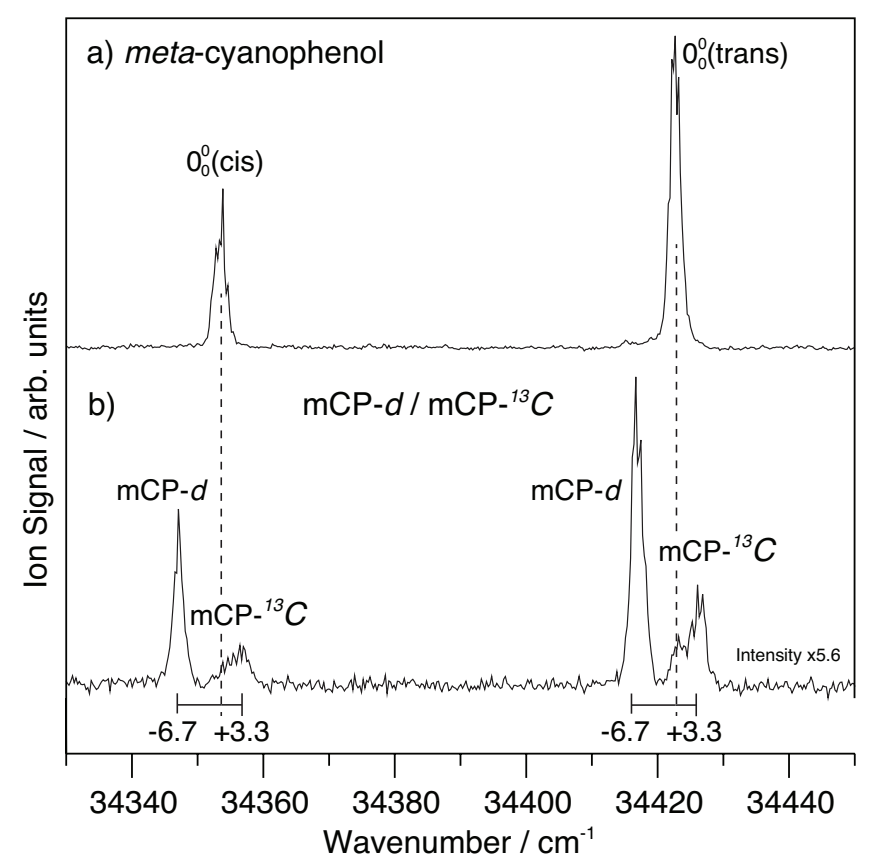

Figure 7: 2C-R2PI spectra of the $0_{0}^{0}$ band region of cis- and trans-meta-cyanophenol. (a) mCP mass channel $(\mathrm{m} / \mathrm{e}=119), \mathrm{b}) \mathrm{mCP}+1$ mass channel $(\mathrm{m} / \mathrm{e}=120)$ showing the signals of ${ }^{13} \mathrm{C}-\mathrm{mCP}$ and $\mathrm{mCP}-d$. 


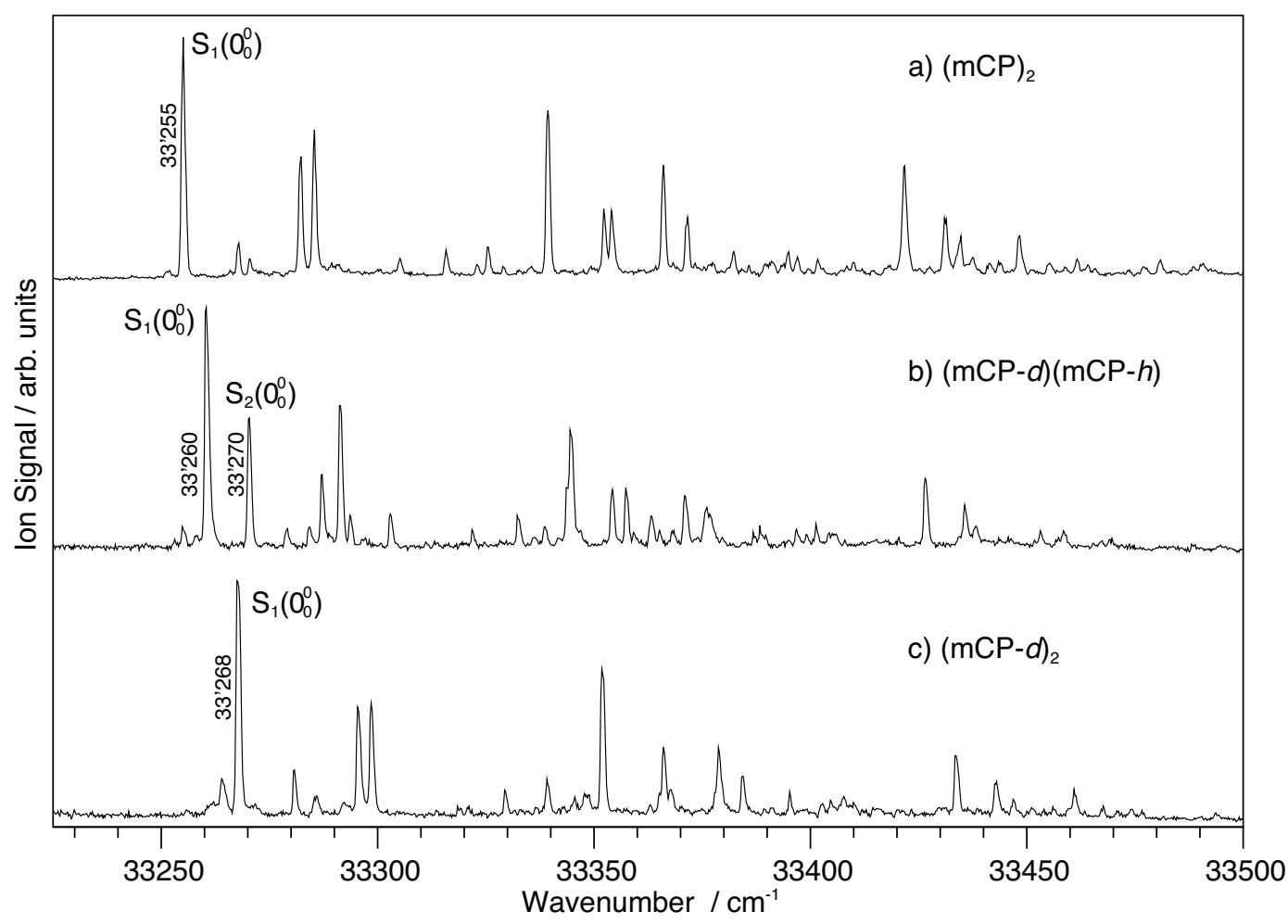

Figure 8: 2C-R2PI spectra of (a) all- $h$ - $(m \text {-cyanophenol })_{2}$, (b) (m-cyanophenol- $\left.h\right)(m$-cyanophenol$d)$ and (c) $(m \text {-cyanophenol- } d)_{2}$. 

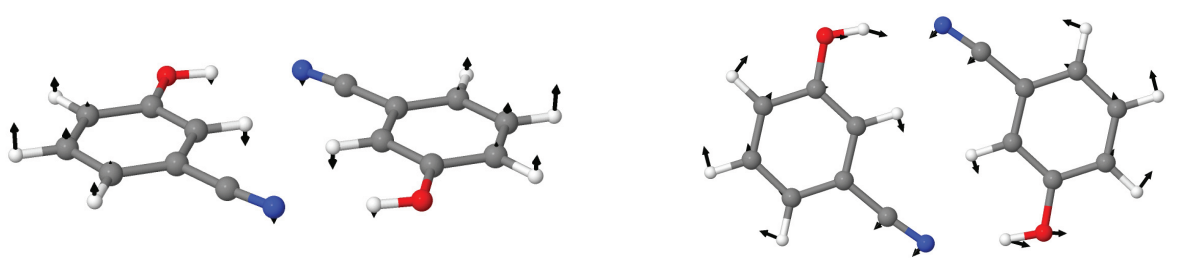

$v_{2}(\beta): 13.0 \mathrm{~cm}^{-1}$

$v_{15}\left(\delta \mathrm{C}-\mathrm{OH}_{\mathrm{as}}\right): 412.1 \mathrm{~cm}^{-1}$
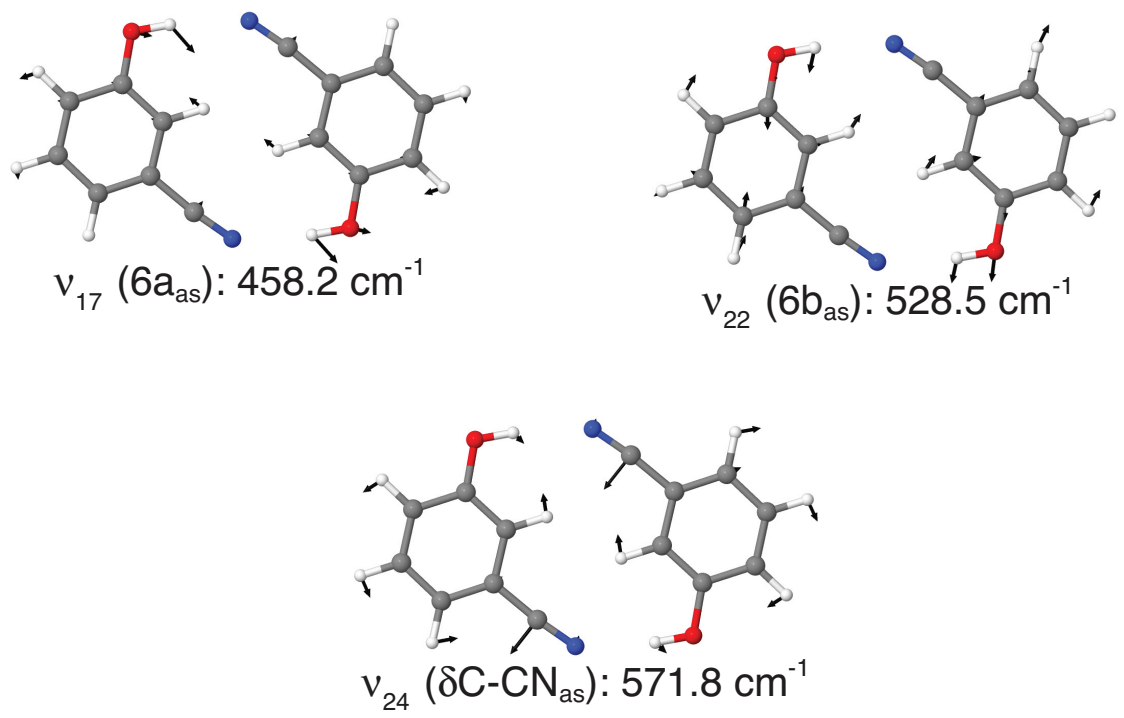

Figure 9: SCS-CC2/aVTZ calculated eigenvectors of the low-frequency antisymmetric $\left(a_{u}\right)$ modes of (m-cyanophenol $)_{2}$. 
1

2

3

4

5

6
7

8

9

10

11

12

13

14

15

16

17

18

19

20

21

22

23

24

25

26

27

28

29

30

31

32

33

34

35

36

37

38

39

40

41

42

43

44

45

46

47

48

49

50

51

52

53

54

55

56

57

58

59

60

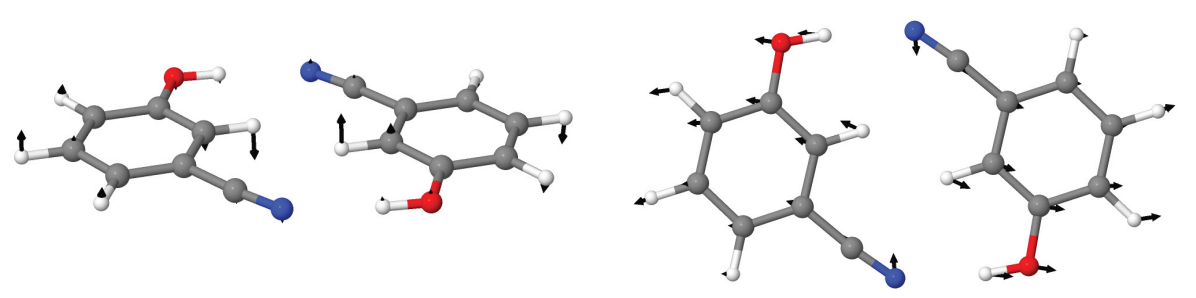

$v_{1}(\delta): 10.8 \mathrm{~cm}^{-1}$

$v_{4}(\sigma): 79.2 \mathrm{~cm}^{-1}$

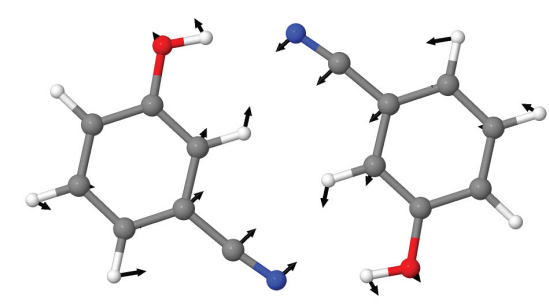

$v_{6}(\chi): 108.5 \mathrm{~cm}^{-1}$

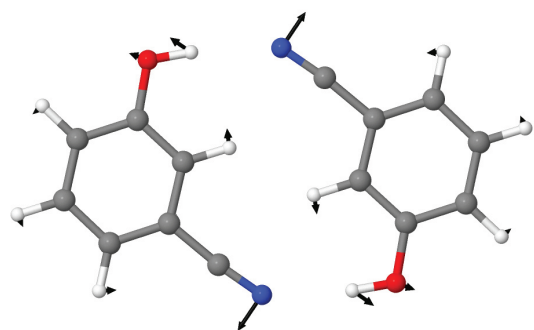

$v_{9}(\delta C C-N): 171.4 \mathrm{~cm}^{-1}$
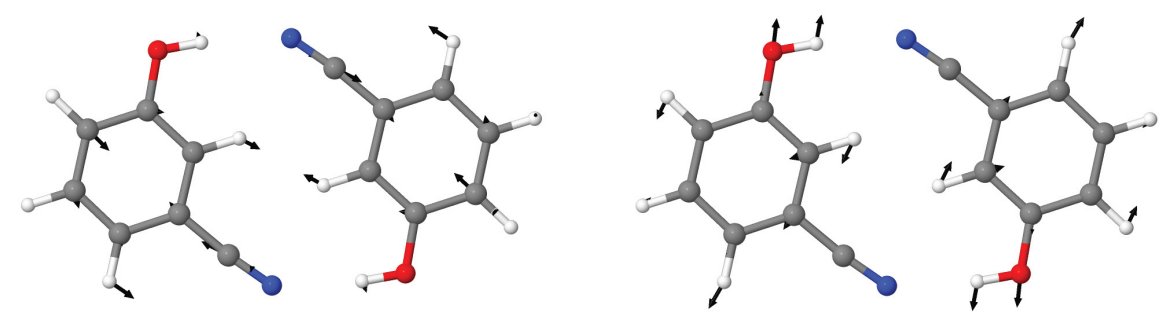

$v_{18}(6 a): 458.2 \mathrm{~cm}^{-1}$

$v_{21}(6 b): 528.4 \mathrm{~cm}^{-1}$

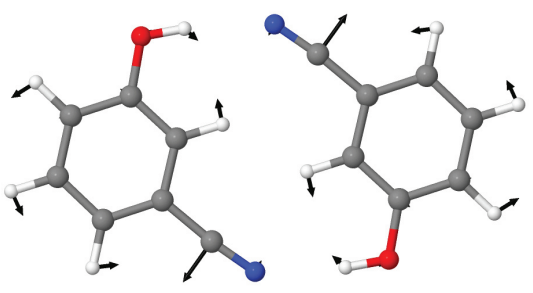

$v_{23}(\delta \mathrm{C}-\mathrm{CN}): 569.4 \mathrm{~cm}^{-1}$

Figure 10: SCS-CC2/aVTZ calculated eigenvectors of the low-frequency totally-symmetric $\left(a_{g}\right)$ modes of $(m \text {-cyanophenol })_{2}$. 

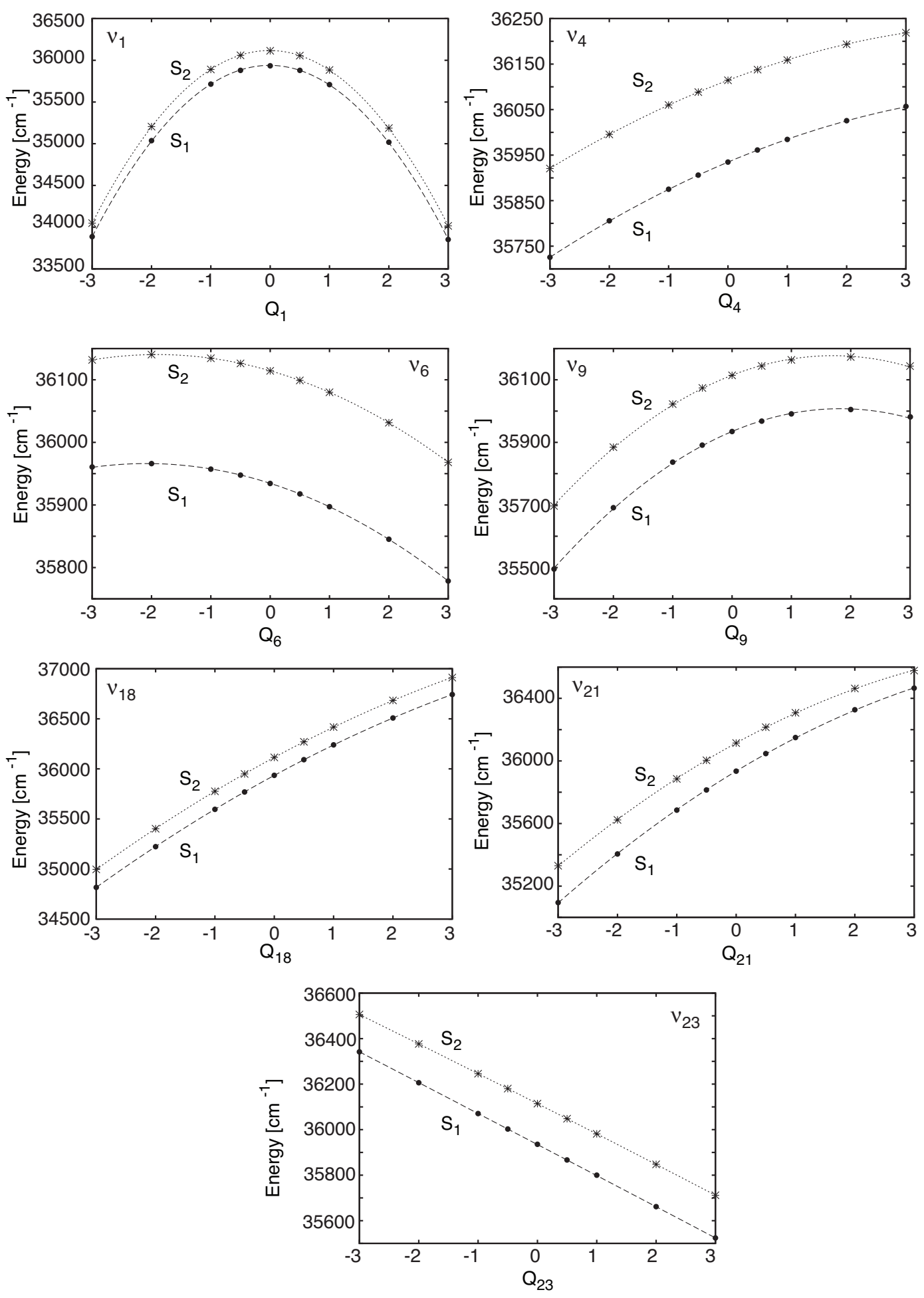

Figure 11: Least-squares fits to the calculated $S_{1}$ and $S_{2}$ excitation energies of ( $m$-cyanophenol $)_{2}$ along the normal-modes $Q_{i}$ of the modes $v_{1}, v_{4}, v_{6}, v_{9}, v_{18}, v_{21}$ and $v_{23}$. The corresponding vibrational eigenvectors are shown in Figs. 10 and fig:vibs-au. The $a b$ initio energies are shown as dots for $S_{1}$ and crosses for $S_{2}$. 

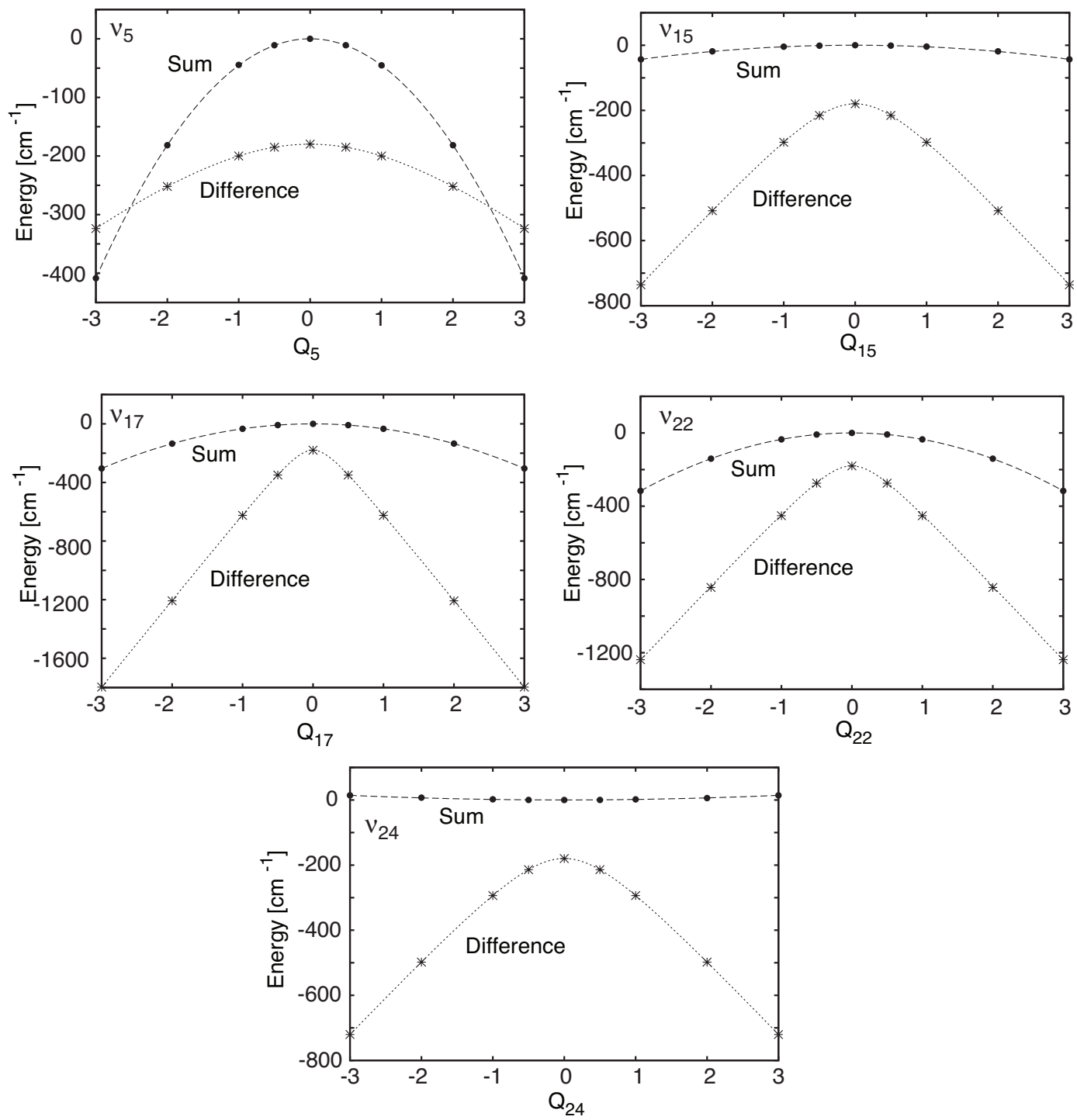

Figure 12: Least-squares fits to the sum (dashed) and difference (dotted) of the excitation energies along the normal-mode eigenvectors of the vibrations $v_{5}, v_{15}, v_{17}$ and $v_{24}$. The ab initio energies are shown as dots for $S_{1}$ and crosses for $S_{2}$. 


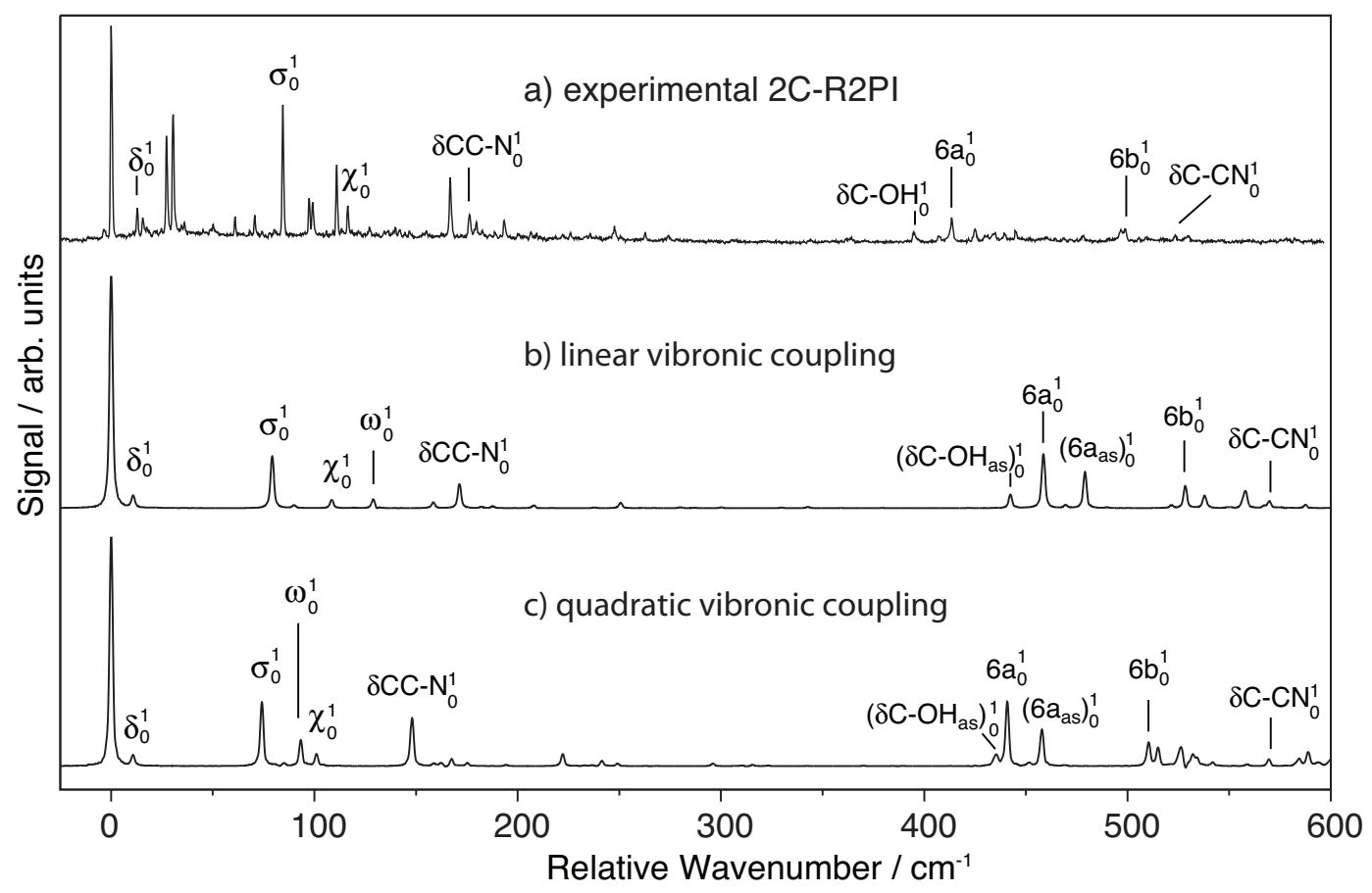

Figure 13: (a) Experimental 2C-R2PI spectrum of $(\mathrm{mCP})_{2}$ compared to the MCTDH simulated spectra based on (b) the LVC and (c) the QVC model. 
2

3

4

5

6

10

11

12

13

14

15

16

17

18

19

20

21

22

23

24

25

26

27

28

29

30

31

32

33

34

35

36

37

38

39

40

41

42

43

44

45

46

47

48

49

50

51

52

53

54

55

56

57

58

59

60

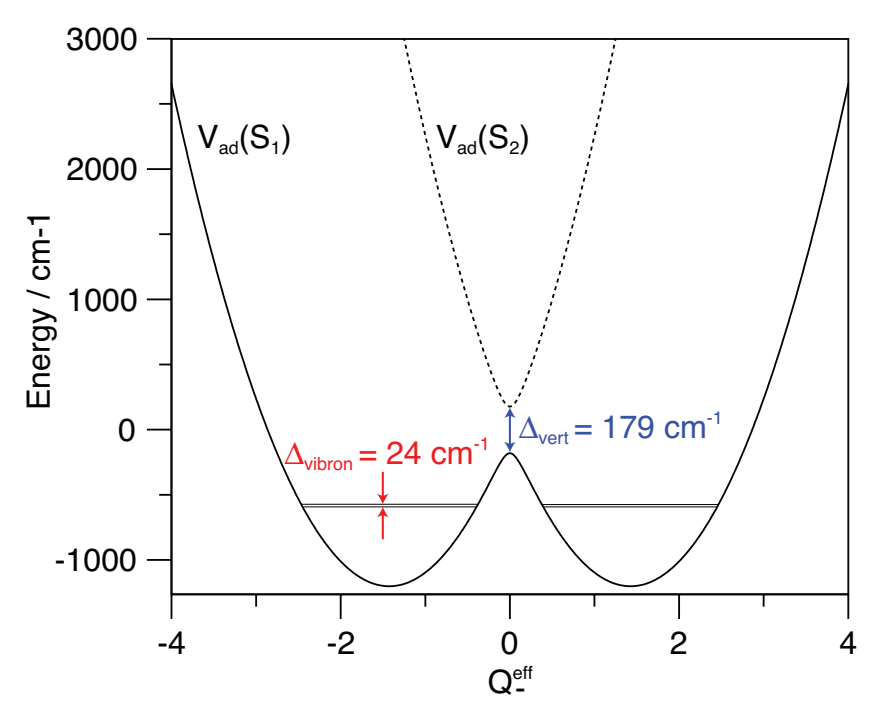

Figure 14: $S_{1}$ and $S_{2}$ excited-state adiabatic potential energy surfaces along the effective mode $\mathrm{Q}_{-}^{\text {eff }}$. The vertical excitation energy splitting $\Delta_{\text {vert }}$ at the ground state equilibrium geometry $Q_{-}^{\text {eff }}=$ 0 is identical to the calculated Davydov splitting $\Delta_{e l}$. 


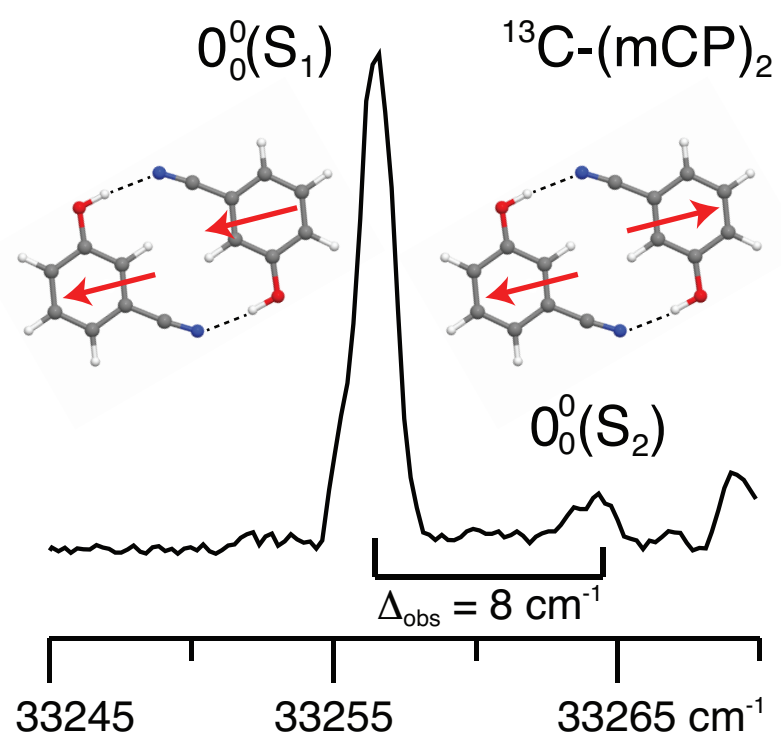

Figure 15: TOC graphic 